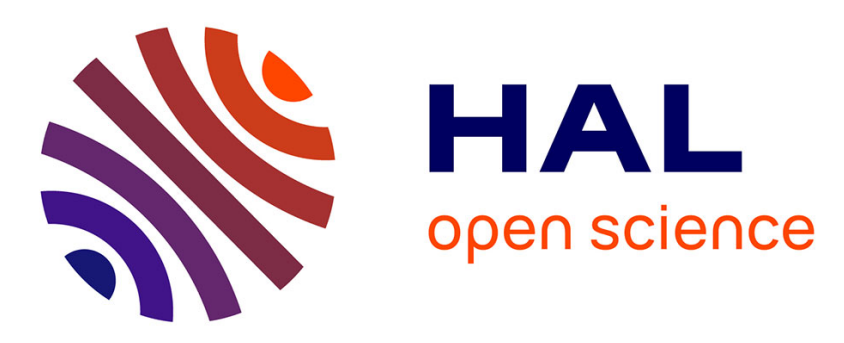

\title{
Unilateral contact induced blade/casing vibratory interactions in impellers: Analysis for rigid casings
}

Alain Batailly, Markus Meingast, Mathias Legrand

\section{To cite this version:}

Alain Batailly, Markus Meingast, Mathias Legrand. Unilateral contact induced blade/casing vibratory interactions in impellers: Analysis for rigid casings. Journal of Sound and Vibration, 2015, 17, pp.244262. 10.1016/j.jsv.2014.10.010 . hal-01120157

\section{HAL Id: hal-01120157 \\ https://hal.science/hal-01120157}

Submitted on 24 Feb 2015

HAL is a multi-disciplinary open access archive for the deposit and dissemination of scientific research documents, whether they are published or not. The documents may come from teaching and research institutions in France or abroad, or from public or private research centers.
L'archive ouverte pluridisciplinaire HAL, est destinée au dépôt et à la diffusion de documents scientifiques de niveau recherche, publiés ou non, émanant des établissements d'enseignement et de recherche français ou étrangers, des laboratoires publics ou privés. 


\title{
Unilateral contact induced blade/casing vibratory interactions in impellers: analysis for rigid casings
}

\author{
Alain Batailly*, Markus Meingast, Mathias Legrand
}

\begin{abstract}
This contribution addresses the vibratory analysis of unilateral-contact induced structural interactions between a bladed impeller and its surrounding rigid casing. Such assemblies can be found in helicopter or small aircraft engines for instance and the interactions of interest shall arise due to the always tighter operating clearances between the rotating and stationary components. The investigation is conducted by extending to cyclically symmetric structures an in-house time-marching based tool dedicated to unilateral contact occurrences in turbomachines. The main components of the considered impeller together with the associated assumptions and modeling principles considered in this work are detailed. Typical dynamical features of cyclically symmetric structures, such as the aliasing effect and frequency clustering are explored in this nonlinear framework by means of thorough frequency-domain analyses and harmonic trackings of the numerically predicted impeller displacements. Additional contact maps highlight the existence of critical rotational velocities at which displacements potentially reach high amplitudes due to the synchronization of the bladed assembly vibratory pattern with the shape of the rigid casing. The proposed numerical investigations are also compared to a simpler and (almost) empirical criterion: it is suggested, based on nonlinear numerical simulations with a linear reduced order model of the impeller and a rigid casing, that this criterion may miss important critical velocities emanating from the unfavorable combination of aliasing and contact-induced higher harmonics in the vibratory response of the impeller. Overall, this work suggests a way to enhance guidelines to improve the design of impellers in the context of nonlinear and nonsmooth dynamics.
\end{abstract}

\section{Keywords}

Nonlinear dynamics, contact mechanics, aeronautical engine, rotor/stator interactions, vibrations.

\section{Interactions générées par les contacts aubes/carter pour un rouet centrifuge: étude avec carters rigides}

\author{
Alain Batailly*, Markus Meingast, Mathias Legrand
}

\begin{abstract}
Résumé
Cet article a pour objet l'analyse vibratoire des interactions structurelles entre un rouet centrifuge et le carter environnant engendrées par des contacts aubes/carter. De telles structures sont notamment utilisées dans les moteurs d'hélicoptères ou les moteurs d'avion de petite taille. L'apparition de contacts entre rouet et carter est la conséquence de jeux de fonctionnement réduits qui permettent d'optimiser le rendement du moteur. L'étude présentée est une extension aux structures à symétrie cyclique d'une stratégie numérique existante et dédiée à la simulations d'interactions aube/carter dans les turbomachines aéronautiques. L'article décrit notamment les différentes parties d'un rouet ainsi que l'ensemble des hypothèses de simulation et des principes de modélisation utilisés. Les caractéristiques des structures à symétrie cyclique, comme le phénomène d'aliasing et la haute densité fréquentielle sont présentés dans le contexte d'une analyse non-linéaire et illustrés dans les domaines fréquentiel et temporel. Des cartes de contact permettent de mettre en évidence le lieu des contacts aube/carter sur la circonfèrence de la surface de contact du carter sur toute une plage de vitesses de rotation. Ces dernières sont complétées par des spectres soulignant la variation des amplitudes de vibration en fonction de la vitesse de rotation. Les résultats des simulations numériques sont confrontés à un critère simplifié de conception; cette confrontation suggère que le critère simplifié ne permet pas de rendre compte de plusieurs zones d'interaction critiques associées à une combinaison défavorable du phénomène d'aliasing avec des super-harmoniques de la réponse vibratoire du rouet. Finalement, le travail présenté permet de proposer uen façon d'améliorer les règles de conception des rouets à l'aide de considérations de dynamique non-linéaire et non régulière.
\end{abstract}

Mots-clés

Dynamique nonlinéaire, mécanique numérique du contact, moteur aéronautique, interaction rotor/stator, vibrations.

Laboratoire de Dynamique des Structures et Vibrations, Université McGill, 817 Sherbrooke Ouest, Pavillon McConnell, Salle 122, H3A-0C3, Montréal, Québec, Canada Auteur à contacter : Tél. : +15143985321; fax : +15143987365. Courriel : alain.batailly@mcgill.ca

\section{Introduction}

Impellers, also known as centrifugal compressors, are found in various industrial turbomachine applications at different scales such as aerospace centrifugal compressors in aeronautical engines, automotive turbochargers and microelectromechanical systems (MEMS) micromotors, to name a few. Impellers offer very high compression ratios and are usually more efficient than axial compressors but shall generate undesired noise [1]. In this regard, a majority of the existing archived publications explores possible ways to reduce the noise generated by impellers [2, 3]. The main interest of the present work lies in the proper characterization of critical rotational velocities where amplitudes of motion of the bladed impeller may reach undesired high levels induced by a unilateral-contact driven mechanism leading to vibratory reso- 
nances. Comparable considerations could not be found in the literature for centrifugal impellers even though unilateral contacts and their effects on the dynamics have already been investigated at the journal/bearing interface for small-scale micro-rotor systems $[4,5]$. Overall, very few investigations have targeted the dynamical responses of such assemblies from the structural standpoint even though structural resonances which shall arise mostly in automotive turbochargers due to very high rotational operating velocities are reported in $[6,7]$.

Structural unilateral and frictional contact interactions correspond to one type of possible nonlinear dynamical couplingusually referred to as rotor/stator interactions [8] — within turbomachines. They are in a vast majority generated due to minimal operating clearances between rotating and stationary components and shall be split into two main categories: (1) contact occurs at the journal/bearing interface $[9,10]$ in supporting devices, or (2) between blade tips and surrounding casings [11, 12]. The present works aims at modeling impeller/casing interactions that may be observed in aeronautical engines and intends to provide a comprehensive analysis of the possible nonlinear mechanisms induced by blade-tip/casing contact interactions for elaborate geometries such as the one depicted in Figure 1. The proposed methodology is also relevant to axial compressors.

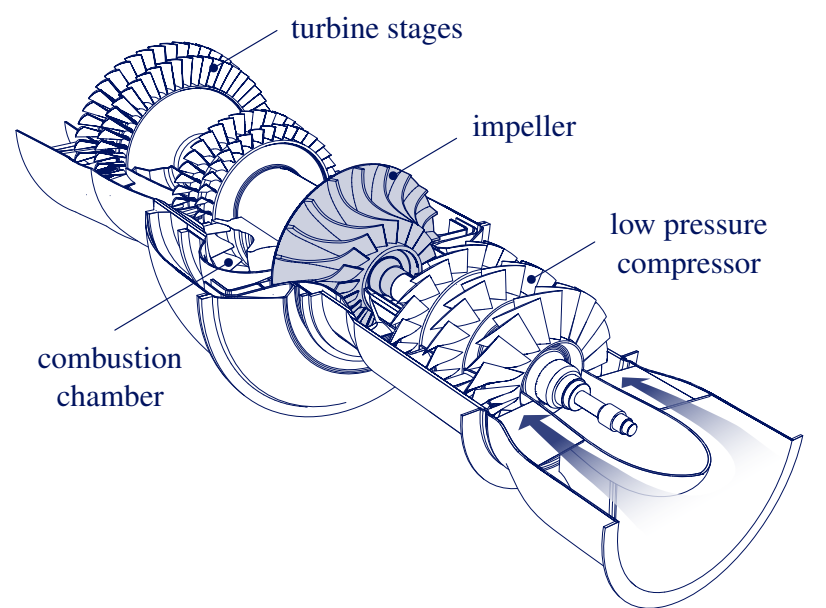

Figure 1. Cut view of an aeronautical engine

Recent experimental investigations [13] highlighted the severity of blade-tip/casing interactions in axial compressors and blade failures were observed. Accordingly, a thorough comprehension of these undesired events is crucial for manufacturers. Numerical developments [12, 14] shed light on the corresponding involved nonlinear dynamics, but for axial compressors only. Impellers feature additional numerical difficulties mainly because of the sophisticated complexity of the blade geometry. Furthermore and contrary to axial compressors, relatively low frequency free vibration modes of impellers usually exhibit disk modes which provoke significant vibratory levels evenly distributed over the entire assembly. Such assemblies are typically blisks which are machined from a single piece of material. Their sensitivity to mistuning $[15,16]$ goes beyond the scope of this study and it is assumed that the impeller is perfectly tuned or cyclically symmetric.

Cyclically symmetric structures support high modal density as well as frequency clustering [17] and adequate post-processing tools in the frequency domain are required to efficiently conduct proper analyses of the dynamics. These post-processing tools are extensively detailed in the paper and include interaction maps obtained through dedicated two-dimensional Fourier transforms, contact areas maps on the casing and systematic harmonic tracking. Additionally, special attention is paid to the existence of super-harmonics in the vibratory response of the impeller as well as their spatial distribution along the circumference: accordingly, focus is also made on the so-called aliasing effect.

As previously mentioned, one distinct feature of centrifugal compressors is the blade-tip geometry and the high curvature-of commonly $90^{\circ}$ - between the inlet and the outlet, as detailed in Figure 2, requires a precise three-dimensional contact detection procedure. Such curvature implies both significant axial and radial deformations as opposed to axial compressors for which axial deformations are commonly ignored. Based on a dedicated explicit time integration procedure [11], a configuration of interest inducing contact without friction between the centrifugal compressor and its surrounding casing is explored. The casing is assumed to be rigidly distorted along a two- or three-nodal diameter shape. The interest mainly lies in the detection of vibratory resonances emerging at rotational velocities said to be critical.

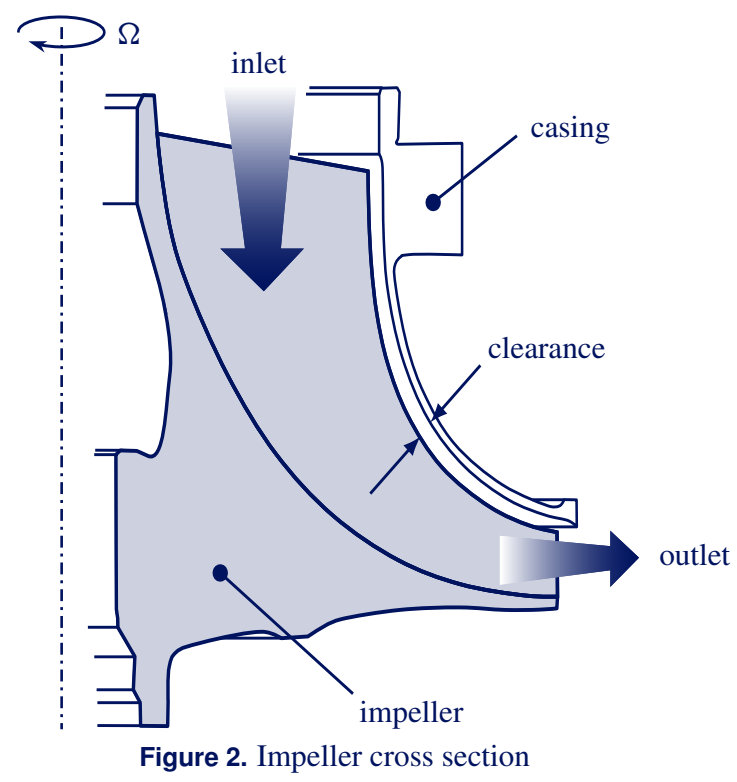

The system is described in the second section of the article. The linear criterion commonly considered for the prediction of interactions is briefly introduced in the third section. The fourth section summarizes the proposed modelling and the methodology employed for the numerical simulations. Associated challenges due to the cyclic symmetry are extensively reviewed in the fifth section. Finally, in the last section, two case studies are proposed for two distinct casing distortions.

Note Amplitudes are normalized with respect to reference data for confidentiality purposes.

\section{Aeronautical engine: main features and as- sumptions}

The present numerical investigation centers on the contact-induced vibratory response of the centrifugal compressor of an aeronautical engine such as the one depicted in Figure 1. The centrifugal compressor is located between the low pressure compressor stages and the combustion chamber of the engine, and advantageously allows for compact dimensions. The assembly includes ten identical main blades and associated splitter blades, as pictured in Figure 3, and is thus recognized as a cyclically symmetric structure with $N=10$ elementary sectors. Based on both ex- 


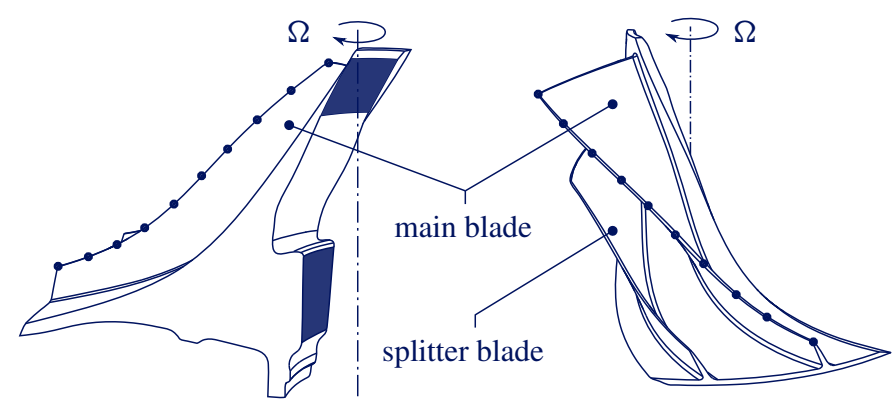

Figure 3. Elementary sector of the impeller

perimental evidence and industrial experience, a few simplifying assumptions are considered to maintain a reasonable computational cost in the simulations:

Axis of rotation The shaft supporting the impeller is assumed to be perfectly rigid. Boundary areas where the impeller is clamped are depicted in red in Figure 3. Additionally, the angular velocity $\Omega$ of the impeller is constant.

Rigidly distorted casing This work extends the methodology proposed in [11] to any structure exhibiting cyclic symmetry. The casing undergoes a static distortion to a rigid configuration which induces unilateral contact. By choice, the deformed casing features two or three privileged evenly distributed contact areas (also named lobes in the remainder) along its circumference.

Blade contact interface Contact essentially occurs at the tip of the main blades while the stiffer splitter blades are not prone to interaction. As a matter of fact, the frequencies of eigenmodes for which splitter blades feature significant amplitudes of vibration are high enough to be neglected in this study. Accordingly, numerical treatment of the contact conditions is handled along the chord of the main blades only [see blue nodes in Figure 3]; ten contact locations along the chord ensure convergence.

\section{Linear criterion for interaction free designs}

Current design guidelines for safe impellers are shortly reminded. The angular velocity is critical when contact interactions between the impeller and the surrounding casing lead to high amplitudes of vibration. Such a condition is typically satisfied when both the impeller and the casing are vibrating along a corresponding free vibration mode. Additionally, identical nodal diameters $n_{\mathrm{d}}$ - see section 4.2 for details - allows geometrically compatible vibratory modeshapes. These two aspects shall be cast as follows:

$f_{\mathrm{i}}^{n_{\mathrm{d}}}(\Omega)= \pm f_{\mathrm{c}}^{n_{\mathrm{d}}} \pm n_{\mathrm{d}} \Omega$

where $f_{\mathrm{i}}^{n_{\mathrm{d}}}(\Omega)$ is the eigenfrequency associated to a $n_{\mathrm{d}}$-nodal diameter free vibration mode of the impeller calculated at $\Omega$ and $f_{\mathrm{c}}^{n_{\mathrm{d}}}$ is the eigenfrequency of a corresponding $n_{\mathrm{d}}$-nodal diameter free vibration mode of the casing. However, the directional friction forces prohibit the impeller from inducing a backward rotating wave on the casing. Similarly, the impeller cannot experience a forward travelling wave due to contact. In the end, the interaction condition (1) becomes:

$f_{\mathrm{i}}^{n_{\mathrm{d}}}(\Omega)=\left|-f_{\mathrm{c}}^{n_{\mathrm{d}}}+n_{\mathrm{d}} \Omega\right|$

Equation (2) does not account for potential super- and sub-harmonics that may arise in the nonlinear dynamics and is accordingly named the linear interaction condition. Currently, condition (2) is the main tool available to designers for the quick prediction of critical velocities. However, recent numerical investigations [18] have emphasized the limitations of this criterion in the context of highly nonlinear interactions initiated by structural unilateral contact occurrences.

\section{Unilateral contact interactions modelling}

\subsection{Finite element discretization}

The finite element model of an elementary sector contains about 120,000 degrees-of-freedom (dof) so that the finite element model of the full impeller involves about a million dof. The mesh comprises 20-node quadratic brick elements and complies with the common industrial standards recommending at least two elements across the blade thickness, three elements along the blade root, and three elements across the disk thickness.

\subsection{Cyclic symmetry}

The impeller of interest is numerically perfectly tuned, i.e. the ten elementary sectors are identical. This cyclic symmetry yields block-circulant mass $\mathbf{M}$ and stiffness $\mathbf{K}$ matrices in polar coordinates:

$\mathbf{Y}=\left[\begin{array}{cccccc}\mathbf{Y}_{0} & \mathbf{Y}_{1}^{\top} & \mathbf{0} & \mathbf{0} & \ldots & \mathbf{Y}_{1} \\ \mathbf{Y}_{1} & \mathbf{Y}_{0} & \mathbf{Y}_{1}^{\top} & \mathbf{0} & \ldots & \mathbf{0} \\ \mathbf{0} & \mathbf{Y}_{1} & \mathbf{Y}_{0} & \mathbf{Y}_{1}^{\top} & \mathbf{0} & \ldots \\ \vdots & & & \ddots & & \\ \mathbf{Y}_{1}^{\top} & \mathbf{0} & \ldots & \mathbf{0} & \mathbf{Y}_{1} & \mathbf{Y}_{0}\end{array}\right], \quad \mathbf{Y}=\mathbf{K}$ or $\mathbf{M}$

where $\mathbf{Y}_{0}$ and $\mathbf{Y}_{1}$ correspond to an elementary sector ${ }^{1}$ and the coupling term between two adjacent sectors, respectively. The Fourier matrix $\mathbf{F}$ block-diagonalizes such matrices as follows [19, 20]:

$\hat{\mathbf{Y}}=\mathbf{F}^{\top} \mathbf{Y} \mathbf{F}=\left[\begin{array}{ccccc}\hat{\mathbf{Y}}^{(0)} & & & & \\ & \hat{\mathbf{Y}}^{(1)} & & \mathbf{0} & \\ & & \hat{\mathbf{Y}}^{(2)} & & \\ & \mathbf{0} & & \ddots & \\ & & & & \hat{\mathbf{Y}}^{\left(\left\lfloor\frac{N}{2}\right\rfloor\right)}\end{array}\right]$

Each block $\hat{\mathbf{Y}}^{\left(n_{\mathrm{d}}\right)}, n_{\mathrm{d}}=0, \ldots,\left\lfloor\frac{N}{2}\right\rfloor$ is called a nodal diameter matrix and refers to a spatial harmonic of the structure [20]. The term nodal diameter is here preferred to spatial harmonic to avoid potential confusion with the time harmonics introduced later. Accordingly, the governing equations of cyclic structures are block-uncoupled in the Fourier space. The free vibration modes of the impeller of interest may exhibit $N_{h}=\left\lfloor\frac{N}{2}\right\rfloor+1=6$ distinct nodal diameter matrices: $\hat{\mathbf{Y}}^{\left(n_{\mathrm{d}}\right)}, n_{\mathrm{d}}=0, \ldots, 5$. The eigenfrequencies of the impeller may be plotted with respect to their associated nodal diameter in a veering diagram as depicted in Figure 4. Eigenmodes of cyclically symmetric structures, such as the ones depicted in Figure 5, are clustered in modal families [20], each consisting of $N$ sector modes of a given pattern: blade bending, blade torsion, disk mode, etc. It has a single $n_{\mathrm{d}}=0$ mode (that is a standing wave) as well as a pair of two modes for every higher nodal diameter ${ }^{2}$.

Each modal family features analogous mode shapes for a given sector: for instance, in the first modal family, the main

\footnotetext{
${ }^{1}$ More details on these matrices are provided in Eq. (5).

${ }^{2}$ With the exception of the nodal family associated to the nodal diameter $n_{\mathrm{d}}=N_{h}-1$ when there is an even number of blades for which there is only
} one mode in the corresponding modal family. 


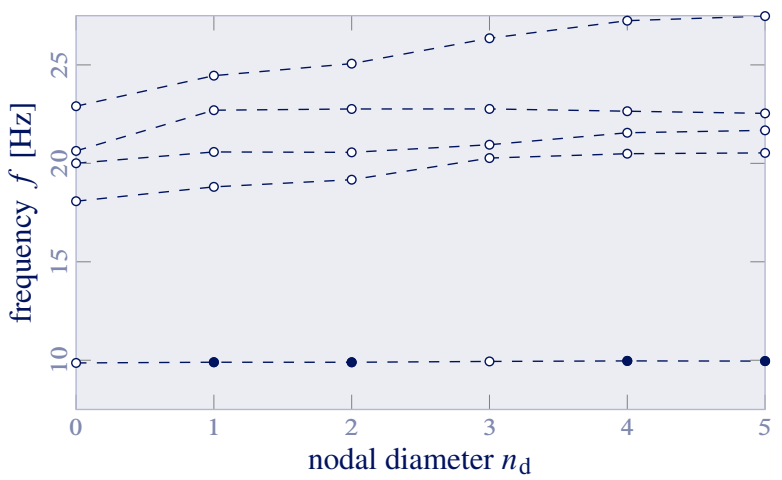

Figure 4. Veering diagram of the first five modal families of the impeller at rest. Modes marked with a blue dot [๑] are displayed in Figure 5


(a) $n_{\mathrm{d}}=1$

(b) $n_{\mathrm{d}}=2$

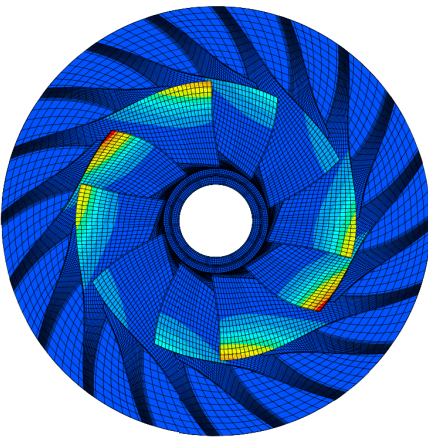

(c) $n_{\mathrm{d}}=4$

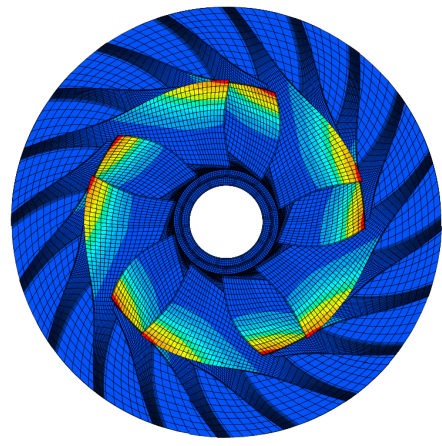

(d) $n_{\mathrm{d}}=5$

Figure 5. Free vibration modes of the first modal family

blades only vibrate along their first bending mode as depicted in Figure 5. For higher modal families, free-vibration modes may involve the splitter blades or the disk as well. As an example, two free-vibration modes of the sixth modal family are pictured in Figure 6 where one can observe non negligible vibration of the disk in the vicinity of the trailing edge. The modes pictured in Figs. 5 and 6 were computed from a computationally efficient reduced model detailed in the following section.

\subsection{Modal synthesis reduction}

Due to its very large size, the finite element model cannot be implemented as such in the contact algorithm. Accordingly, is employed a component mode synthesis technique based on an extension [21] of the Craig-Bampton method [22] which embeds centrifugal stiffening in the reduced order model (ROM) directly computed in the Fourier basis [20]. The procedure requires three stiffness matrices calculated at three distinct angular velocities: $\mathbf{K}(0), \mathbf{K}\left(\Omega_{\max } / 2\right)$ and $\mathbf{K}\left(\Omega_{\max }\right)$ as well as the mass matrix $\mathbf{M}$ of an elementary sector:

1. Matrix reorganization: rows and columns of $\mathbf{Y}_{s}$ are sorted

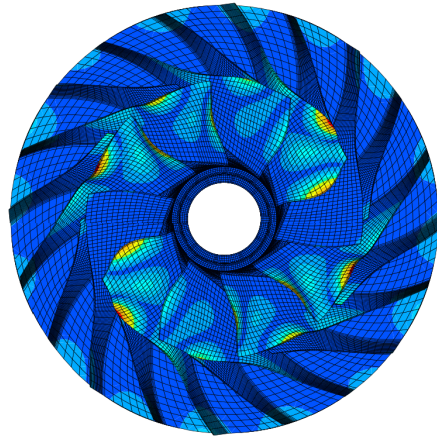

(a) $n_{\mathrm{d}}=2$

Figure 6. Two free vibration modes of the sixth modal family

as follows:

$$
\mathbf{Y}_{s}=\left[\begin{array}{ccc}
\mathbf{Y}_{i i} & \mathbf{Y}_{i b} & \mathbf{Y}_{i c} \\
\mathbf{Y}_{b i} & \mathbf{Y}_{b b} & \mathbf{Y}_{b c} \\
\mathbf{Y}_{c i} & \mathbf{Y}_{c b} & \mathbf{Y}_{c c}
\end{array}\right] \text { and } \mathbf{u}=\left(\begin{array}{l}
\mathbf{u}_{i} \\
\mathbf{u}_{b} \\
\mathbf{u}_{c}
\end{array}\right)
$$

with:

$\mathbf{Y}_{0}=\left[\begin{array}{ll}\mathbf{Y}_{i i} & \mathbf{Y}_{i b} \\ \mathbf{Y}_{b i} & \mathbf{Y}_{b b}\end{array}\right]$ and $\mathbf{Y}_{1}=\left[\begin{array}{ll}\mathbf{Y}_{i c} & \mathbf{0}_{n_{i}, n_{i}-n_{c}+n_{b}} \\ \mathbf{Y}_{b c} & \mathbf{0}_{n_{b}, n_{i}-n_{c}+n_{b}}\end{array}\right]$

where $\mathbf{Y}=\mathbf{K}$ or $\mathbf{M}$ and vectors $\mathbf{u}_{i}, \mathbf{u}_{b}$, and $\mathbf{u}_{c}$ respectively stand for the internal, boundary, and cyclic nodal displacements as pictured in Figure 7.

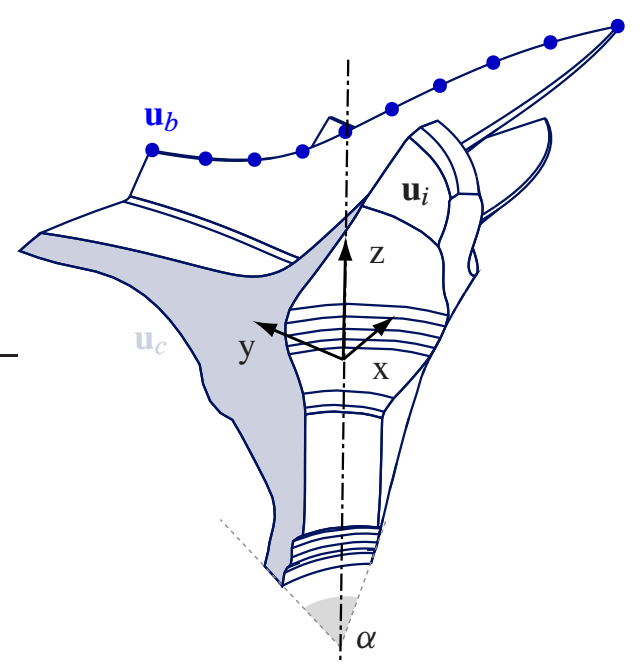

Figure 7. Partition of the nodes of an elementary sector

2. Computation of the nodal diameter matrices: each nodal diameter matrix is computed from the finite element matrices as follows [20]:

$$
\hat{\mathbf{Y}}^{\left(n_{\mathrm{d}}\right)}=\left[\begin{array}{cc}
\mathbf{Y}_{0}+\left(\mathbf{Y}_{1}+\mathbf{Y}_{1}^{\top}\right) \cos \left(n_{\mathrm{d}} \alpha\right) & \left(\mathbf{Y}_{1}-\mathbf{Y}_{1}^{\top}\right) \sin \left(n_{\mathrm{d}} \alpha\right) \\
\left(\mathbf{Y}_{1}^{\top}-\mathbf{Y}_{1}\right) \sin \left(n_{\mathrm{d}} \alpha\right) & \mathbf{Y}_{0}+\left(\mathbf{Y}_{1}+\mathbf{Y}_{1}^{\top}\right) \cos \left(n_{\mathrm{d}} \alpha\right)
\end{array}\right]
$$

where $\alpha$ is the angular span of a sector as depicted in Figure 7.

3. Centrifugal stiffening: the three stiffness matrices accounting for centrifugal stiffening [21] are expressed as:

$$
\begin{aligned}
\hat{\mathbf{K}}_{0}^{\left(n_{\mathrm{d}}\right)} & =\hat{\mathbf{K}}_{s}^{\left(n_{\mathrm{d}}\right)}(0) \\
\hat{\mathbf{K}}_{1}^{\left(n_{\mathrm{d}}\right)} & =\frac{1}{3 \Omega_{\max }^{2}}\left(16 \hat{\mathbf{K}}_{s}^{\left(n_{\mathrm{d}}\right)}\left(\frac{\Omega_{\max }}{2}\right)-\hat{\mathbf{K}}_{s}^{\left(n_{\mathrm{d}}\right)}\left(\Omega_{\max }\right)-15 \hat{\mathbf{K}}_{s}^{\left(n_{\mathrm{d}}\right)}(0)\right) \\
\hat{\mathbf{K}}_{2}^{\left(n_{\mathrm{d}}\right)} & =\frac{4}{3 \Omega_{\max }^{4}}\left(\hat{\mathbf{K}}_{s}^{\left(n_{\mathrm{d}}\right)}\left(\Omega_{\max }\right)-4 \hat{\mathbf{K}}_{s}^{\left(n_{\mathrm{d}}\right)}\left(\frac{\Omega_{\max }}{2}\right)+3 \hat{\mathbf{K}}_{s}^{\left(n_{\mathrm{d}}\right)}(0)\right)
\end{aligned}
$$


4. Modal reduction: for each of the three considered values of $\Omega$, the modal reduction basis $\hat{\boldsymbol{\Phi}}_{\eta}^{\left(n_{\mathrm{d}}\right)}(\Omega)$ is:

$\hat{\boldsymbol{\Phi}}^{\left(n_{\mathrm{d}}\right)}(\Omega)=\left[\begin{array}{cc}\mathbf{I} & \stackrel{\mathbf{0}}{\longrightarrow} \\ \hat{\boldsymbol{\Psi}}_{\mathrm{s}}^{\left(n_{\mathrm{d}}\right)}(\Omega) & \hat{\boldsymbol{\Psi}}_{\mathrm{co}}^{\left(n_{\mathrm{d}}\right)}(\Omega)\end{array}\right]$

in which $\eta$ constraint modes are computed for every angular velocity, all stored column-wise in $\hat{\boldsymbol{\Psi}}_{\text {co }}^{\left(n_{\mathrm{d}}\right)}(\Omega)$. For each harmonic, the global reduction basis $\hat{\Upsilon}^{\left(n_{\mathrm{d}}\right)}$ may be then rearranged as:

$\hat{\boldsymbol{\Upsilon}}^{\left(n_{\mathrm{d}}\right)}=\left[\begin{array}{cc}\mathbf{I} & \mathbf{0} \\ \hat{\boldsymbol{\Psi}}_{\mathrm{s}}^{\left(n_{\mathrm{d}}\right)}(0) & \boldsymbol{\Psi}^{\left(n_{\mathrm{d}}\right), *}\end{array}\right]$

where $\Psi^{\left(n_{\mathrm{d}}\right), *}$ contains an orthonormal basis generated from the static and constraint modes given in Eq. (8). The computation of the reduced harmonic mass and stiffness matrices is expressed as:

$$
\begin{aligned}
& \mathbf{K}_{0, r}^{\left(n_{\mathrm{d}}\right)}=\hat{\boldsymbol{\Upsilon}}^{\left(n_{\mathrm{d}}\right), \top} \hat{\mathbf{K}}_{0}^{\left(n_{\mathrm{d}}\right)} \hat{\boldsymbol{\Upsilon}}^{\left(n_{\mathrm{d}}\right)} \\
& \mathbf{K}_{1, r}^{\left(n_{\mathrm{d}}\right)}=\hat{\boldsymbol{\Upsilon}}^{\left(n_{\mathrm{d}}\right), \top} \hat{\mathbf{K}}_{1}^{\left(n_{\mathrm{d}}\right)} \hat{\boldsymbol{\Upsilon}}^{\left(n_{\mathrm{d}}\right)} \\
& \mathbf{K}_{2, r}^{\left(n_{\mathrm{d}}\right)}=\hat{\Upsilon}^{\left(n_{\mathrm{d}}\right), \top} \hat{\mathbf{K}}_{2}^{\left(n_{\mathrm{d}}\right)} \hat{\boldsymbol{\Upsilon}}^{\left(n_{\mathrm{d}}\right)} \\
& \mathbf{M}_{r}^{\left(n_{\mathrm{d}}\right)}=\hat{\boldsymbol{\Upsilon}}^{\left(n_{\mathrm{d}}\right), \top} \hat{\mathbf{M}}^{\left(n_{\mathrm{d}}\right)} \hat{\Upsilon}^{\left(n_{\mathrm{d}}\right)}
\end{aligned}
$$

5. $\Omega$-dependent reduced-order model: the final reduced matrices become:

$$
\begin{aligned}
& \mathbf{K}_{r}(\Omega)=\mathbf{F}^{\top} \operatorname{Bdiag}\left(\left[\mathbf{K}_{0, r}^{\left(n_{\mathrm{d}}\right)}+\Omega^{2} \mathbf{K}_{1, r}^{\left(n_{\mathrm{d}}\right)}+\Omega^{4} \mathbf{K}_{2, r}^{\left(n_{\mathrm{d}}\right)}\right]_{, n_{\mathrm{d}}=0, \ldots,\left\lfloor\frac{N}{2}\right\rfloor}\right) \mathbf{F} \\
& \mathbf{M}_{r}(\Omega)=\mathbf{F}^{\top} \operatorname{Bdiag}\left(\left[\mathbf{M}_{r}^{\left(n_{\mathrm{d}}\right)}\right]_{, n_{\mathrm{d}}=0, \ldots,\left\lfloor\frac{N}{2}\right\rfloor}\right) \mathbf{F}
\end{aligned}
$$

where Bdiag refers to a block-diagonal matrix:

$\operatorname{Bdiag}\left(\left[\mathbf{A}^{n_{\mathrm{d}}}\right]_{\left., n_{\mathrm{d}}=0, \ldots, i\right]}\right)=\left[\begin{array}{llll}\mathbf{A}^{0} & & & \\ & \mathbf{A}^{1} & & \\ & & \ddots & \\ & & & \mathbf{A}^{i}\end{array}\right]$

Matrices (10) are computed only once. They are multiple orders of magnitude smaller in size than the original finite element matrices. The stiffness matrix $\mathbf{K}_{r}(\Omega)$ of the reduced model is simply denoted $\mathbf{K}_{r}$ in the remainder.

\subsection{Modal analysis and convergence}

The degrees of freedom at the impeller-shaft interface are clamped as depicted in Figure 3. The associated Campbell diagram is displayed in Figure 8 in the relative frame.

It shows the first two modal families of the impeller. The ten eigenfrequencies associated with the first modal family are clustered around $f=10$ while the ten eigenfrequencies of the second modal family are more clearly distinguishable between $f=18$ and $f=20.5$ at rest.

As pictured in Figure 9, $\eta=150$ leads to a satisfactory compromise between accuracy and computational cost since results obtained for higher $\eta$ are almost perfectly superimposed. The corresponding error between the first 50 eigenfrequencies of the ROM and the their counterparts extracted from the full model is less than $1 \%$. The computed ROM contains 900 dof-300 physical dof for contact treatment and 600 component modes-which is about $0.09 \%$ of the model full size. A similar convergence analysis has been carried out over the full angular velocity range in order to confirm the robustness of the proposed approach; asymptotic convergence is also achieved in time.

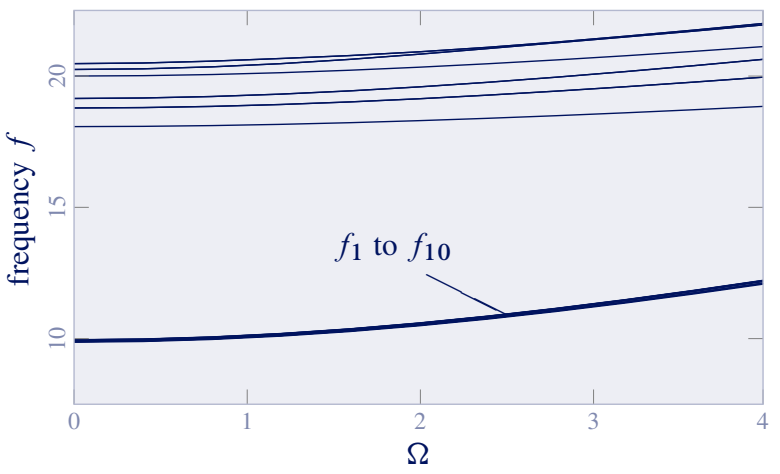

Figure 8. Campbell diagram in the relative frame

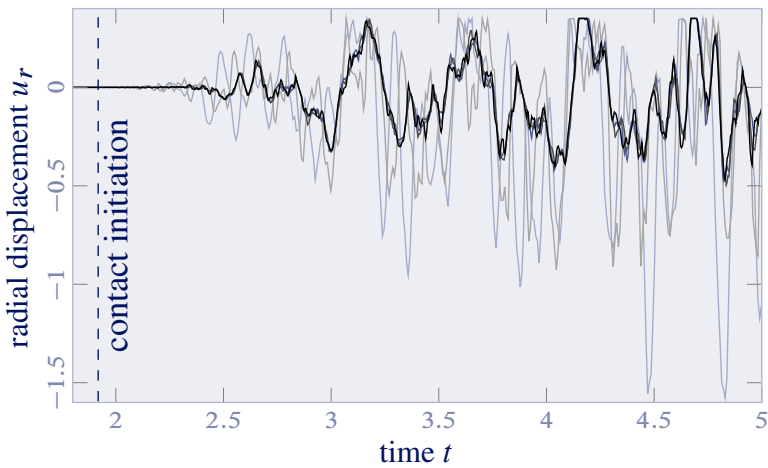

Figure 9. Convergence plot of the radial displacement: $\eta=50(-)$, $\eta=100(\sqcup), \eta=150(\longleftarrow), \eta=200(\sqcup)$, and $\eta=250(\square)$

\subsection{Unilateral contact conditions}

The time-domain solution method presented in [11] is briefly recalled for the sake of clarity. Friction is not accounted for.The procedure involves the explicit central differences scheme combined with a Lagrange multiplier-based contact algorithm [23] implemented on the ten contact interface nodes per sector. At each time step $q+1$, the procedure is divided into three steps:

1. Prediction at time step $q+1$ of the displacements $\mathbf{u}_{r}$ :

$\mathbf{u}_{r}^{q+1, p}=\left[\frac{\mathbf{M}_{r}}{h^{2}}+\frac{\mathbf{D}_{r}}{2 h}\right]^{-1}\left(\left(\frac{2 \mathbf{M}_{r}}{h^{2}}-\mathbf{K}_{r}\right) \mathbf{u}_{r}^{q}+\left(\frac{\mathbf{D}_{r}}{2 h}-\frac{\mathbf{M}_{r}}{h^{2}}\right) \mathbf{u}_{r}^{q-1}\right)$

2. Determination of the gap function and detection of the blade contacting nodes.

3. Correction of the predicted displacements to cancel the predicted penetrations:

$$
\mathbf{g}^{q+1}=\mathbf{C}_{\mathbf{N}}^{\top} \mathbf{u}_{r}^{q+1, c}+\mathbf{g}^{p}=\mathbf{0}
$$

Lagrange multipliers, i.e. contact forces, and updated displacements are:

$$
\left\{\begin{array}{l}
\lambda=\left(\mathbf{C}_{\mathbf{N}}^{\top}\left[\frac{\mathbf{M}_{r}}{h^{2}}+\frac{\mathbf{D}_{r}}{2 h}\right]^{-1} \mathbf{C}_{\mathbf{N}}\right)^{-1} \mathbf{g}^{p} \\
\mathbf{u}_{r}^{q+1}=\mathbf{u}_{r}^{q+1, p}+\left[\frac{\mathbf{M}_{r}}{h^{2}}+\frac{\mathbf{D}_{r}}{2 h}\right]^{-1} \mathbf{C}_{\mathbf{N}} \lambda
\end{array}\right.
$$

\section{Analysis method}

As explained in section 3, there is a need for a more comprehensive approach for the determination of critical angular velocities of the impeller. Beside its restriction to linear considerations, fulfilling condition (2) may not result in effective high vibratory amplitudes. Based on the aforementioned theoretical and numerical developments, the systematic detection of critical angular velocities is now established. 


\subsection{Challenging aspects}

Concerning the numerical predictions of nonlinear interactions in modern turbomachines, the greatest challenge lies in the validation of the results with existing experimental data or industrial evidence. The unaffordable cost of turbomachinery full-scale experiments combined with the hurdle to properly simulate modal interactions makes it particularly arduous for manufacturers to acquire meaningful data. To the authors' knowledge, only a very few publications with detailed experimental results on full-scale engines are available [13], and no data could be found in relation with impellers. Consequently, no attempt to correlate the results presented in this article to experimental data has been undertaken. However, the proposed contact treatment has been numerically validated in a previous work [11] and consistent results with industrial data have been obtained in the context of a single blade/casing interaction within axial compressors [12].

The investigated system also features additional attributes such as frequency clustering and the aliasing effect: both are described in the following subsections.

\subsubsection{Frequency clustering}

As mentioned in section 4.4, the first ten eigenfrequencies of the bladed assembly correspond to the first leading edge bending mode of the main blade and are clustered around $f=10$. This phenomenon is named frequency clustering and is a well-known property of cyclically symmetric structures [17]. Consequently, a Fourier transform of time domain displacements may exhibit closely agglomerated peaks of vibration for a given frequency and modal participations shall not be accurately identified. This

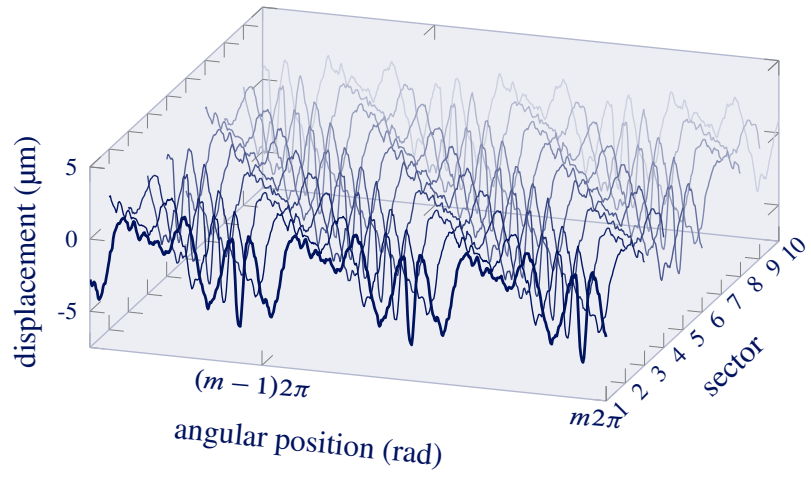

Figure 10. Steady-state response of the ten sectors: main blade leading edge radial displacement (the angular position of the bladed disk is used instead of time for the sake of clarity)

frequency clustering issue may be efficiently tackled by taking advantage of the structure spatial periodicity. Owing to the geometry of a cyclic structure, a steady state response will be periodic both in the time and in the space domains. This is exemplified through a 2-lobe interaction: the impeller response on all sectors, see Figure 10, is transformed to obtain the two-dimensional spectrum in Figure 11. For the example of interest, significant amplitudes of vibration are detected on even nodal diameters only: $n_{\mathrm{d}}=0,2$, and 4 . Clustered vibration peaks on a regular one-dimensional spectrum are, in fact, associated with distinct nodal diameters that can be distinguished with an appropriate $2 \mathrm{D}$ Fourier transform.

\subsubsection{Forced-response aliasing effect}

Two continuous sine waves of order $n$ and $N-n$ and sampled at $N$ points cannot be distinguished: this mathematical feature is termed aliasing. In general, there will be coupling between orders

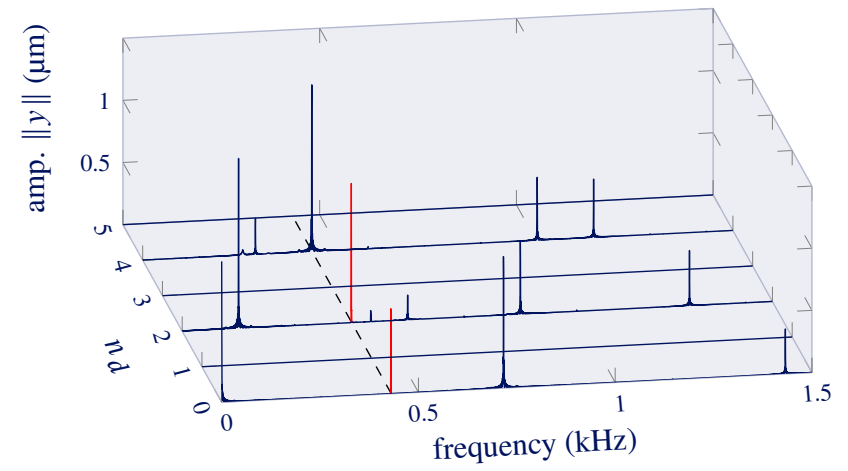

Figure 11. Two-dimensional Fourier transform of the impeller response. Red peaks on nodal diameters 0 and 2 along the dashed line are fictitious and added to illustrate the frequency clustering phenomenon

$n, N-n, N+n, 2 N-n, 2 N+n, \ldots$. A similar situation arises for a $N$-blade assembly externally forced by a sinus wave in space. The potential high harmonics will be aliased by the sampling generated by the $N$ blades. This is illustrated in Figs. 12(a) and 12(b) where two continuous external forcing functions $\cos (n \theta)$, with $n=6$ and $n=8$ respectively, act on $N=10$ evenly spaced blades located at $\theta=2 k \pi / N, k=1, \ldots, N$. When $n=6$, then $N-n=4$ and the forcing is seen by the blades as the function $\cos (4 \theta)$. Since the free-vibration modes of a cyclically

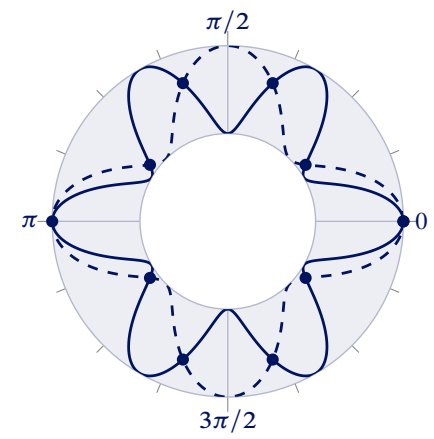

(a) waves $\cos (6 \theta)$ and $\cos (4 \theta)$

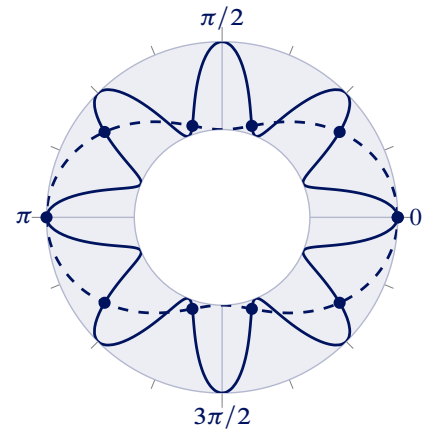

(b) waves $\cos (8 \theta)$ and $\cos (2 \theta)$
Figure 12. Aliasing effect: continuous external forcing waves and its corresponding sampled counterpart $\bullet$ acting on the blades

symmetric structure exhibit a finite number of nodal diameters (see section 4.2), any forcing acting on the structure along high spatial harmonics will be aliased back onto the actual nodal diameters of the structure. With $N=10$ sectors, the impeller free vibration modes feature $n_{\mathrm{d}}=0, \ldots, N_{h}-1$ nodal diameters and any forcing of the form $F \cos (n \theta)$ with $n>N_{h}-1$ will be reflected as a spatial harmonic $\cos (m \theta)$ with $m \in\left[0 ; N_{h}-1\right]$.

\subsubsection{Space-time coupling with a rigid casing}

If the impeller rotates at angular velocity $\Omega$ within a rigid casing statically deformed on $N_{l}$ lobes-meaning $N_{l}$ forced contact areas - each blade will be in contact with the casing probably $N_{l}$ times per revolution. Indeed, because of the significant amplitude of the casing deformation and the fact that the casing is perfectly rigid, potential sub-harmonics are neglectedThus, the fundamental excitation frequency of the external forcing due to contact is given by

$f=N_{l} \Omega$

thereby coupling the spatial pattern $N_{l}$ and the fundamental frequency of the contact forcing $f$. This indicates that the contact- 
induced external forcing on the impeller is a time-dependent forcing with frequency $N_{l} \Omega$ and a space-dependent forcing with frequency $N_{l}$. In the following, the term engine order, denoted $\mathrm{EO}$ refers to the time harmonics of this contact forcing. EO $j$ corresponds to the time harmonic of frequency $j f, j \in \mathbb{N}$. Because of the coupling between time and space induced by the rigid casing, the aliasing effect applies to the space harmonics of the contact forcing and sum up to the graph depicted in Figure 13. Collectively, this is equivalent to the modal appropriation equation:

$\alpha N \pm n_{\mathrm{d}}=k N_{l}, \quad \alpha \in \mathbb{N}, \quad k \in \mathbb{N}^{*}, \quad n_{\mathrm{d}} \in\left[0 ; N_{h}-1\right]$

where $k N_{l}$ is the expected EO on which significant amplitudes of vibration may be observed.

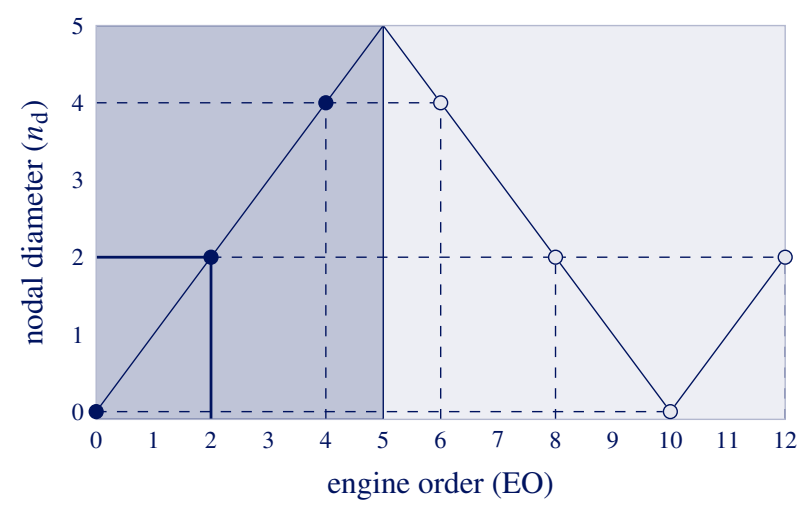

Figure 13. Aliasing effect for $N_{h}-1=5$ : nodal diameters in terms of engine order for a 2-lobe fundamental EO ( - ) and the aliasing effect on its higher harmonics (-- -)

\subsubsection{Case study}

Consider the contact force corresponding to the displacements plotted in Figure 10 for $N_{l}=2$. The contact force-purely radial in this article since no friction is accounted for-on the leading edge of sector 1 main blade is pictured in Figure 14. The asso-

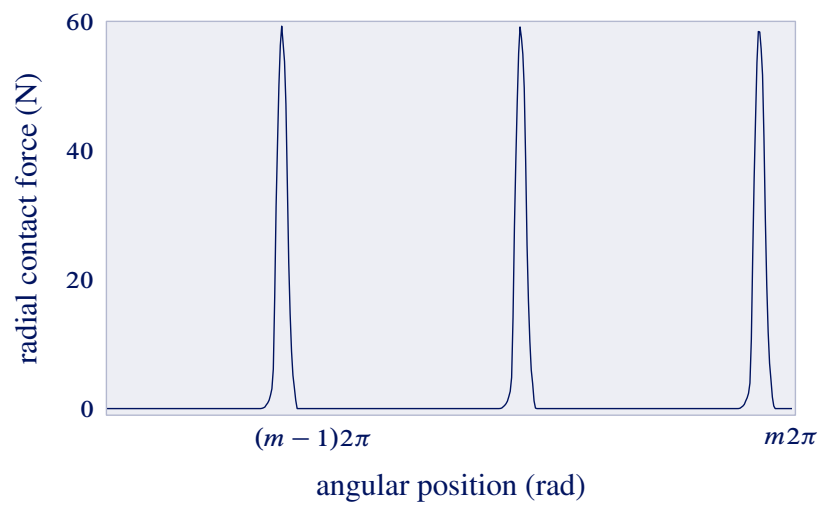

Figure 14. Radial contact force on sector 1 main blade leading edge once steady state is established

ciated spectrum in the frequency domain, in Figure 15, reveals significant peaks of vibration for frequencies $f=k N_{l} \Omega$. The fundamental harmonic is $f=N_{l} \Omega$ in agreement with Eq.(16). The engine orders of interest are $\mathrm{EO} j$ for $j=k N_{l}=2 k$, $k \in \mathbb{N}$. Additionally, the 2D Fourier transform of the space-time contact force computed using the contact force extracted from all separate sectors is displayed in Figure 16. The spectra obtained for each nodal diameter are plotted in Figure 17 where even nodal

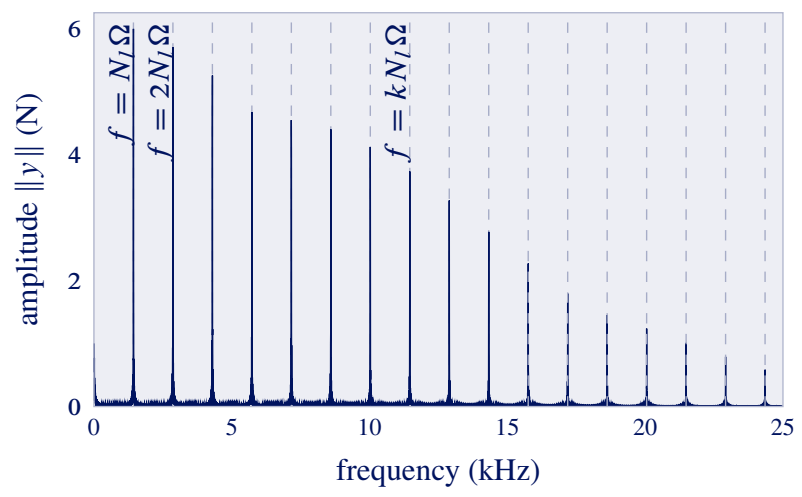

Figure 15. Fourier transform of the contact force depicted in Figure 14

diameters only feature significant amplitude of vibrations: this is in agreement with the fact that all even engine orders are aliased back onto even nodal diameters, as depicted in Figure 13.

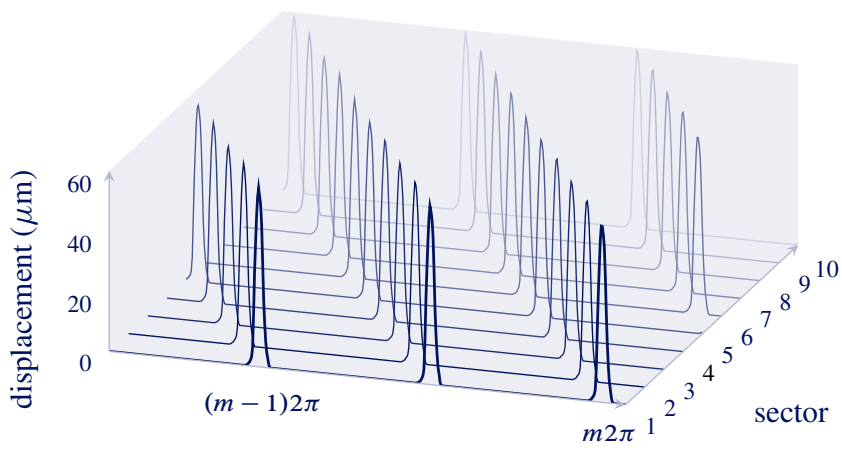

angular position (rad)

Figure 16. Steady-state radial contact force on the ten leading edges (the angular position of the bladed disk is used instead of time for clarity)

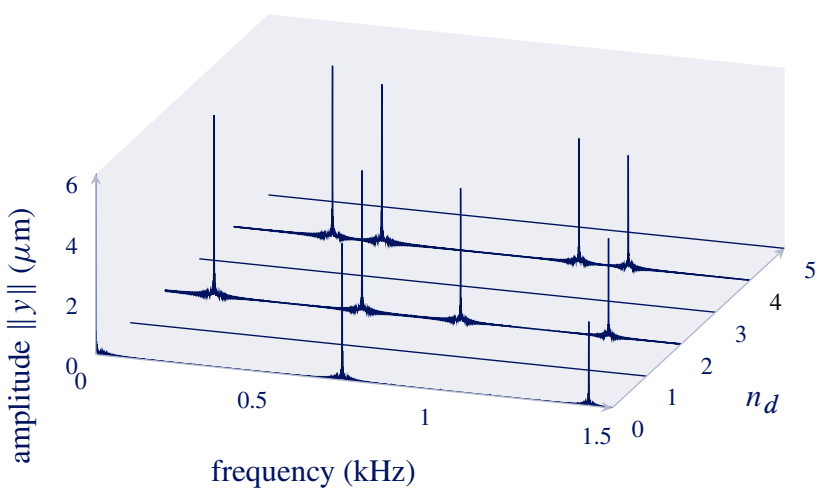

Figure 17. 2D Fourier transform of the radial contact force

\subsection{Detection of critical angular velocities}

Monitoring both vibration and stress levels within blades may be beneficial in order to prevent crack initiation and maximize impeller lifespan. The employed numerical methodology provides straightforward access to the blade-tip displacements. Displacement and stress fields within the full finite element model are thus easily retrieved using the reduction basis given in section 4.3. Supplementary interaction and contact areas maps may also supply useful insight on the impeller dynamics as well as the angular velocities for which maximum stress levels in the structure are reached. 


\subsubsection{Interaction maps}

As previously mentioned, unilateral contact interactions are highly nonlinear and maximum vibration levels are thus nonlinear functions of $\Omega$. In order to identify critical angular velocities, a four-step procedure in the frequency domain is performed:

1. A contact configuration is defined with $N_{l}$ lobes on the casing and an evenly distributed set of angular velocities are considered within the range $\Omega \in\left[\Omega_{\min } ; \Omega_{\max }\right]$;

2 . For each angular velocity $\Omega$, explicit time-stepping simulations are run over a sufficiently long interval of time to reach steady state;

3. Fourier transforms of the calculated displacements are computed for every $\Omega$ and organized in a waterfall diagram similar to the one in Figure 18(a);

4. Vibratory amplitudes are displayed as color maps ranging from blue/low to red/high amplitude as depicted in Figure 18(b).

Subsequent response frequencies involving significant amplitudes are graphically captured. The location of these peaks may then be compared with the crossing of engine order lines $f=k \Omega$ and modal lines $f(\Omega)$ for which the linear criterion (2) predicts interactions.

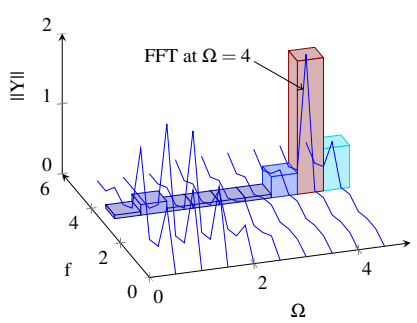

(a)

Figure 18. Contact simulation post-processing tools. Engine order lines are displayed as white lines: (a) waterfall diagram, (b) interaction map

\subsubsection{Contact areas maps}

Relevant information concerning the interaction also involve the locations on the casing of the contact occurrences. A particular attention is paid to the following questions:

- Are the contact areas evolving from one revolution to the next one?

- How long are the contact occurrences or, equivalently, how wide are the associated contact areas on the casing?

- Does a vibratory synchronization arise at angular velocities for which the blades repeatedly always impact the casing at the same locations?

To answer these questions, the casing circumference is divided into 200 identical areas associated to a corresponding counter. When one area is impacted by the blade of the sector of interest, its counter is incremented. The counter array is then converted into a grayscale colour code, from white (no contact) to dark blue (highly impacted area) pictured in the form of a contact map in Figure 19 for the first sector. The contact areas are evidenced by the contrast for a given $\Omega$. A gray column indicates that contact is neither localized nor repetitive. On the contrary, a clear contrast between white and dark blue areas shows that the blade systematically rubs with the casing at the same location. Six dark areas are clearly distinguishable in area 1 in Figure 19: a spatial synchronization occurs between the blade vibration within

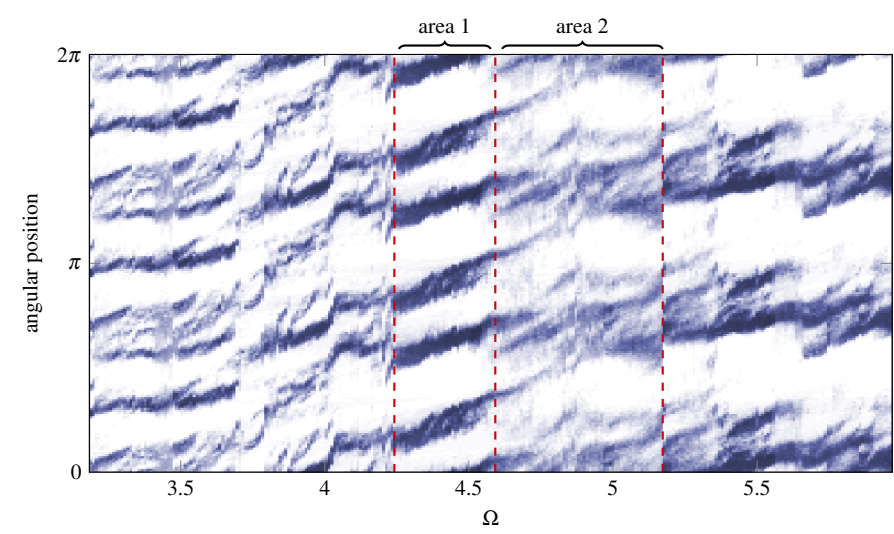

Figure 19. Contact areas map of the first sector of the impeller

the distorted casing. Opposite conclusions may be drawn from the grey areas observed in area 2. One interesting feature of the contact areas maps are the abrupt transitions from one area to a neighbour and an analogy may be drawn with bifurcation diagrams where the transition between one type of solution to another one is similarly sudden. In the context of the targeted application, the clear physical interpretation of the contact maps is attractive.

\subsubsection{Harmonic tracking}

Equation (16) shows that a coupling is expected between spatial patterns $N_{l}$ and the excitation frequency $f$. As mentioned above, obvious considerations and relationships between time (engine orders) and space (nodal diameters) only stand for a limited number $N_{l}$ of lobes on the casing as well as for the fundamental harmonics of the vibratory response of the impeller. The aliasing phenomenon must be accounted for in more sophisticated configurations and the interaction map in section 5.2.1 allows for a clarification of the relationship between engine orders and nodal diameters but misses insight on the modal participations. Consequently, an harmonic tracking procedure able to target a given mode as well as a full set of modes for a given nodal diameter is implemented. A 2D-Fourier transform of the sector responses is carried out to highlight the participating harmonics, see Figs. 20(a) and 20(b). The harmonic tracking procedure follows the amplitude associated to a given point $\left(n_{\mathrm{d}}, \mathrm{EO}\right)$ - for instance, the height of a bar in Figs. 20(a) or 20(b) - with respect to $\Omega$ and two trackings are suggested:

Nodal diameters tracking Tracking of the amplitude of the nodal diameters in the response for a given EO (mean amplitude of the six bars for a given EO in Figure 20(a)).

Engine order tracking Tracking of each nodal diameter $n_{\mathrm{d}}$ for a range of EO (mean amplitude of the first six bars for a given $n_{\mathrm{d}}$ in Figure 20(b)).

\section{Application: unilateral contact interaction be- tween a flexible impeller and a rigidly distorted casing}

The proposed contact configurations involve the two casing profiles depicted in Figure 21: $N_{l}=2$ and $N_{l}=3$. Industrial observations suggest that a two-lobe deformation may stem from a thermal gradient in the structure while the three-lobe configuration is assumed to reflect unexpected: manoeuvre loads. 


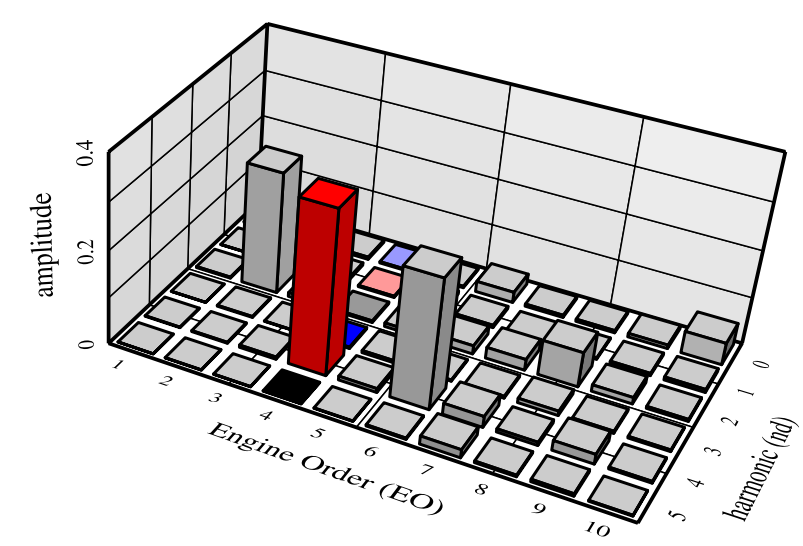

(a)

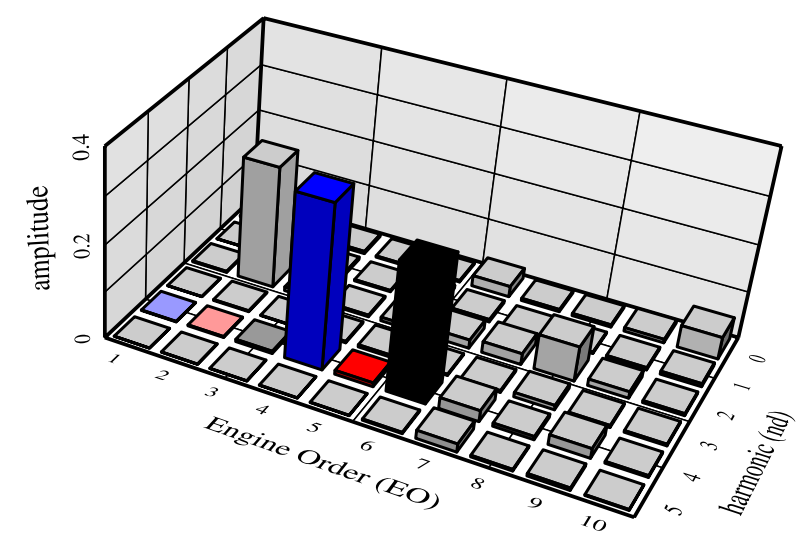

(b)

Figure 20. Blade response 2D-Fourier transform at $\Omega=5.2$ : (a) $n_{\mathrm{d}}$ tracking for $\mathrm{EO}=4$, (b) EO tracking for $n_{\mathrm{d}}=4$

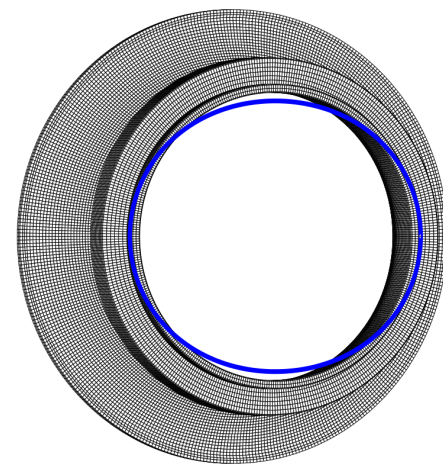

(a)

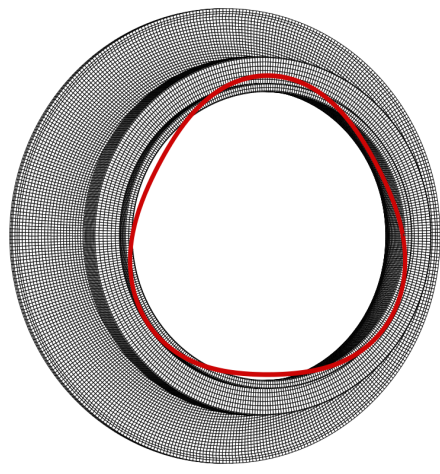

(b)
Figure 21. Simplified representation of the casing distortions over its finite element mesh, (a) 2-lobe deformation, (b) 3-lobe deformation

\subsection{Two-lobe interaction}

When the casing is ovalized, Figure 21(a), there are two privileged contact areas along the circumference of the casing. An interaction map is constructed from the Fourier spectrum of the predicted impeller steady-state response.

\subsubsection{Interaction maps}

Following the procedure described in section 5.2.1, the interaction map associated to the six nodal diameters of the impeller is depicted in Figure 22(a): a major participation of nodal diameters $n_{\mathrm{d}}=2$ and $n_{\mathrm{d}}=4$ is found, as opposed to a minor participation of $n_{\mathrm{d}}=1, n_{\mathrm{d}}=3$ and $n_{\mathrm{d}}=5$. Indeed, the dominant blue colour of the interaction maps associated with odd nodal
diameters-Figs. 22(c), 22(e) and 22(g) — highlights that no significant participation is found throughout the angular velocity range. The addition of the amplitudes in Figs. 22(b) to 22(g) yields the interaction map in Figure 22(a).

Because of the two privileged contact areas, a blade of the impeller is in contact with the casing at least twice per revolution and the expected fundamental engine order is $\mathrm{EO}=2$. Had the number of privileged contact areas been higher than $N_{h}-1$, the fundamental engine order would be obtained through Figure 13. Surprisingly, the highest amplitudes in Figure 22 are located along EO $=6$ which can only be associated with the fourth nodal diameter $n_{\mathrm{d}}=4$, see Figure 22(f).

The fact that the fundamental EO and the EO supporting the highest amplitudes are distinct brings to light the nonlinear nature of the interaction. This partly explains why the linear interaction condition given in Eq. (2) is unable to predict most interactions. Additionally, this example shows that the impeller shall mostly exhibit a two- and four-nodal diameter response within an ovalized casing. Also, since the impeller response is a nonlinear contribution of several linear modes, the concept of nonlinear normal modes [24] may be suitable for an improved comprehension of the dynamics.

\subsubsection{Displacement and stress fields}

Full three-dimensional displacement and stress fields can be retrieved from the time-domain reduced-order response. Exemplary, displacement fields with unilateral contact occurring on the leading edge are displayed in Figs. 23(a) and 23(b), respectively for the blades and the disk. The associated stress fields are pictured in Figs. 23(c) and 23(d). To a symmetric two-lobe contact configuration on the casing corresponds a symmetric displacement field on the impeller. Maximum stress or displacement at a critical

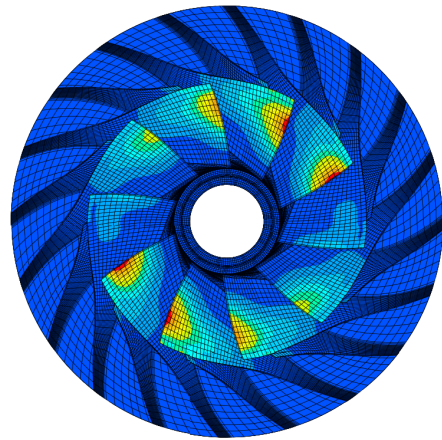

(a) displacement field (blades)

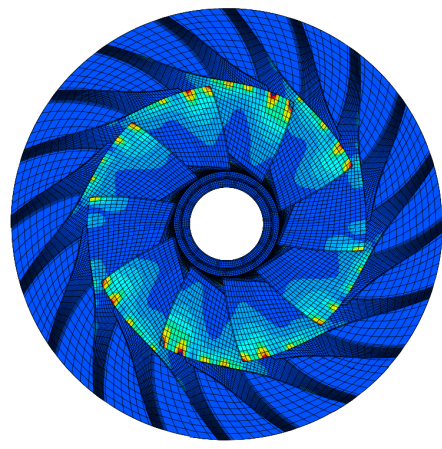

(c) stress field (blades)

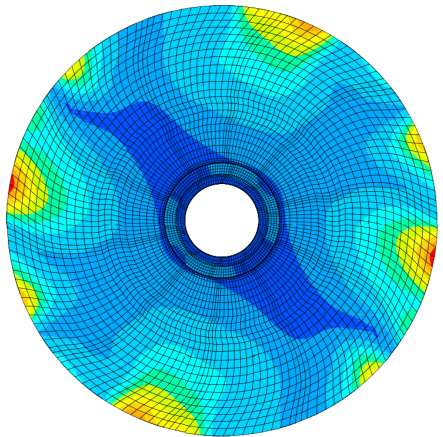

(b) displacement field (disk)

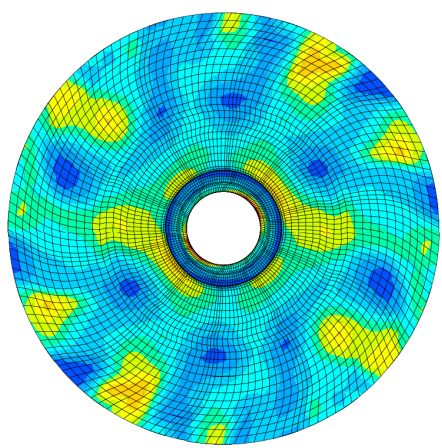

(d) stress field (disk)
Figure 23. Displacement and stress fields at a given time step of a contact simulation with a 2-lobe casing distortion (colour fields are adjusted in order to highlight displacements and stresses for the disk) 


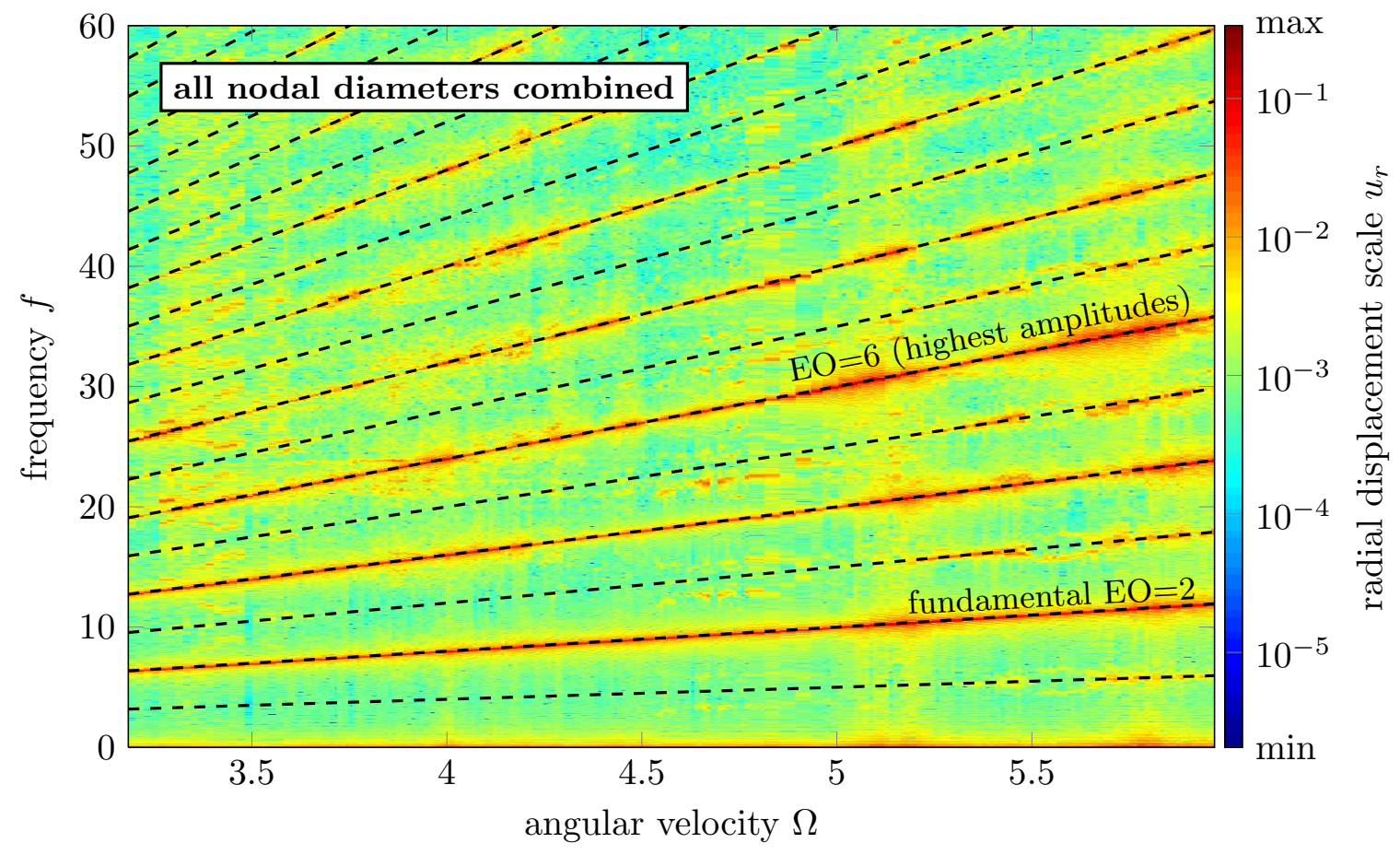

(a)
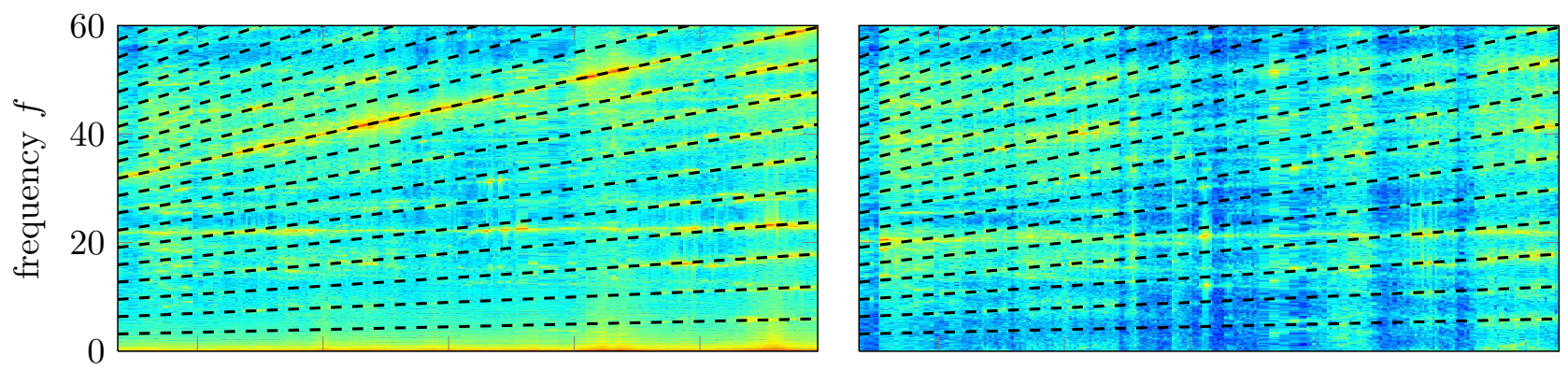

(b)

(c)
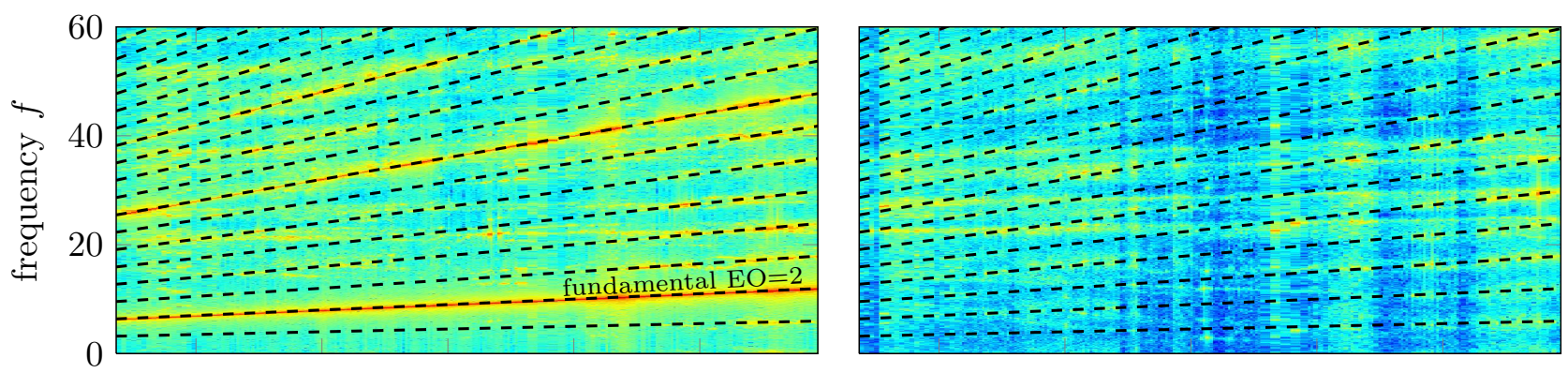

(d)

(e)
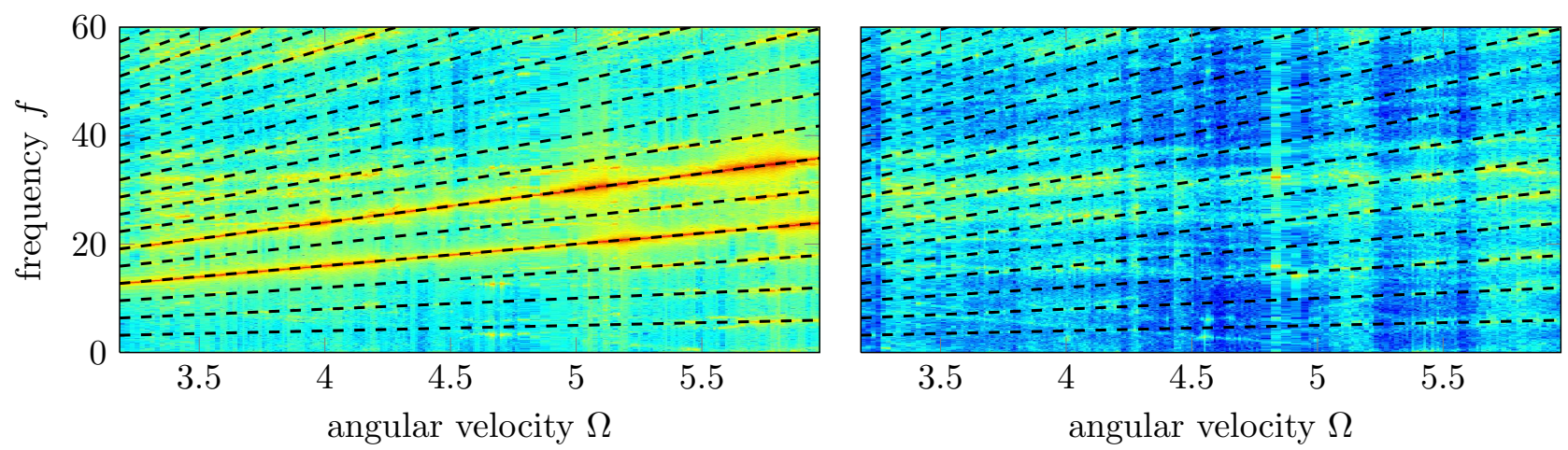

(f)

(g)

Figure 22. Interaction maps for a 2-lobe casing distortion, (a) interaction map, (b) $n_{\mathrm{d}}=0$, (c) $n_{\mathrm{d}}=1$, (d) $n_{\mathrm{d}}=2$, (e) $n_{\mathrm{d}}=3$, (f) $n_{\mathrm{d}}=4$, (g) $n_{\mathrm{d}}=5$ 
location within the impeller can thus be obtained and used, for instance, as a criterion for a design optimization procedure. The implementation of such methodology is of particular industrial relevance [25] in the context of designing robust structures with respect to structural contact constraints for aeronautical engines.

\subsubsection{Contact areas maps}

Interaction maps emphasize the dominant EO and the associated spatial harmonics $n_{\mathrm{d}}$. However, they do not provide any information on the actual contact locations. A prescribed distortion of the casing does not necessarily yield an obvious relationship between the impact cartography and the corresponding impeller dynamics: a dominant four-nodal diameter free vibration mode does not imply four contact areas along the casing circumference. Contact areas maps are pictured in Figs. 24(a) and 24(b) for the

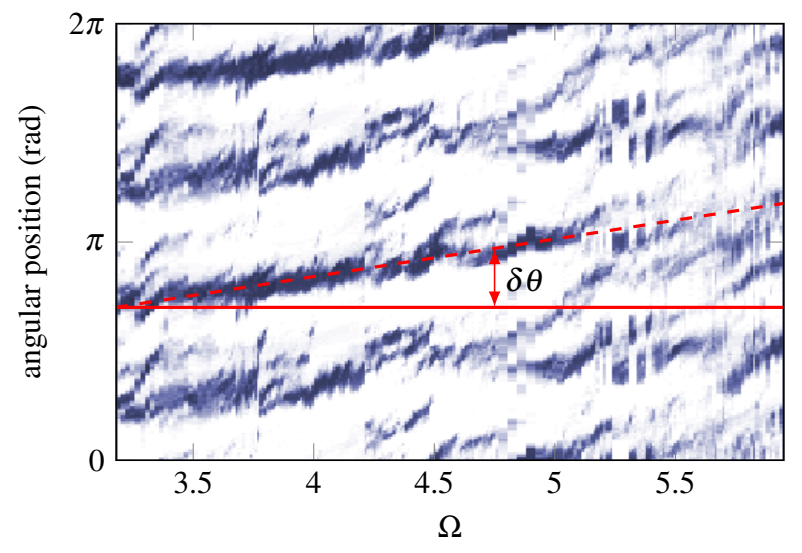

(a)

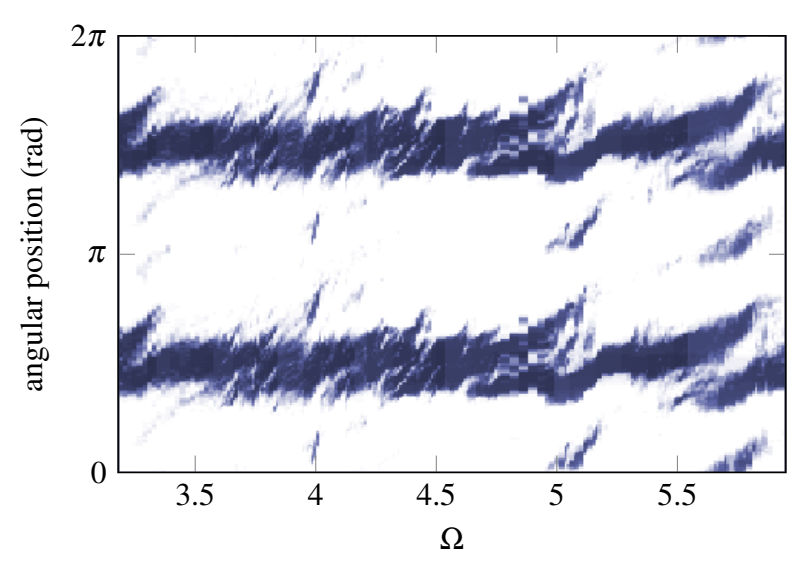

(b)

Figure 24. Contact areas maps. Line (- - ) illustrates the angular shift of the contact location with $\Omega$, (a) leading edge, (b) trailing edge

leading edge and the trailing edge of one sector, respectively.

Interestingly, the two maps largely differ: the blade leading and trailing edges exhibit distinct contact patterns within intricate dynamics. On the leading edge, where the blade is longer and less stiff, about four contact areas are captured for most angular velocities. On the contrary, the stiffer trailing edge is only in contact with the casing twice per revolution throughout most of the angular velocity range. More contact areas are only found for a few narrow angular velocity ranges - such as $\Omega \in[3.97 ; 4]$, $\Omega \in[5 ; 5.2]$ and $\Omega \in[5.65 ; 5.85]$ — which correspond exactly to the highest amplitudes detected in Figure 22(a). As depicted in Figure 24(b), the blade impacts the casing four and six times per revolution, respectively, which matches the peaks of resonance detected along EO $=4$ and $\mathrm{EO}=6$ in Figure 22.
It is remarkable that the angular position of the contact areas between the casing and the leading edge is shifted as the angular velocity is increased, see Figure 24(a). In the considered scenario, the operating clearances are tighter at the trailing edge where contact is initiated. The subsequent wave in the impeller propagates at wave speed $v$ and the delay required to reach the leading edge is approximately $\delta t \simeq l_{b} / v$ where $l_{b}$ denotes the blade chord length. This delay only depends on the material properties of the impeller and the corresponding angular shift $\delta \theta$ is given by $\delta \theta=\delta t \Omega$. The red dotted line in Figure 24(a) illustrates the above simple derivations. Its perfect superimposition with the angular position of the contact areas confirms that the contact locations angular shift linearly depends on $\Omega$.

\subsubsection{Harmonic tracking}

The harmonic tracking procedure from section 5.2.3 is used to clarify possible relationships between the first engine orders $(\mathrm{EO}=1$ to $\mathrm{EO}=6)$ and the impeller nodal diameters. Both nodal diameter and engine order are tracked with respect to $\Omega$. Results are summarized in Figs. 25 and 26.

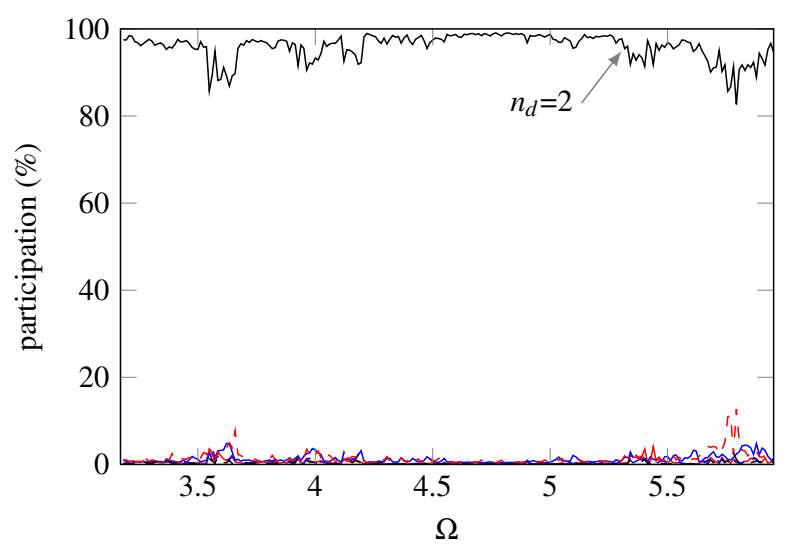

(a)

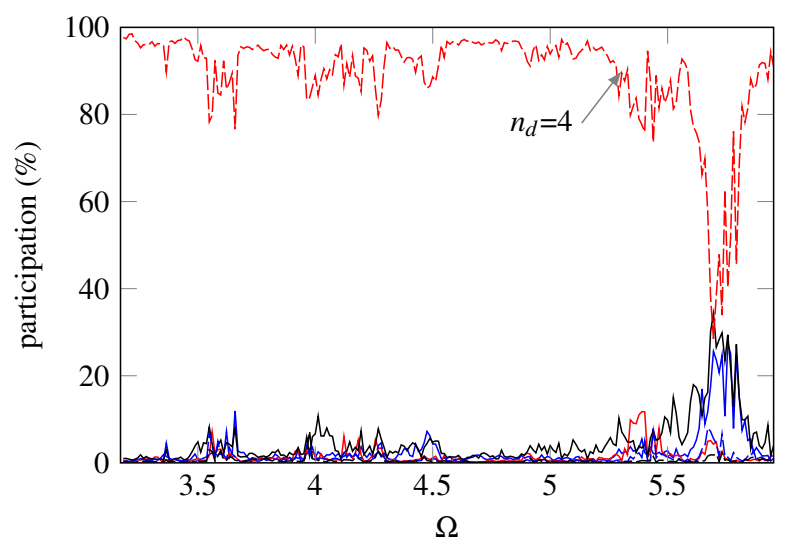

(b)

Figure 25. Nodal diameter tracking: $n_{\mathrm{d}}=0(-), n_{\mathrm{d}}=1(-)$, $n_{\mathrm{d}}=2(-), n_{\mathrm{d}}=3(---), n_{\mathrm{d}}=4(---)$ and $n_{\mathrm{d}}=5(---)$, (a) $\mathrm{EO}=2$, (b) $\mathrm{EO}=4$

Figure 25 shows that along the two dominant $\mathrm{EO}=2$ and $\mathrm{EO}=4$, the vibratory energy is essentially confined in two nodal diameters, $n_{\mathrm{d}}=2$ and $n_{\mathrm{d}}=4$. This is consistent with the interaction maps in Fig 22.

The engine order tracking for $n_{\mathrm{d}}=2$ depicts a major nodal diameter 2 along $E O=2$. Figures 25(a) and 26(a) collectively emphasize the strong internal association, which is also apparent in Figure 22(f), between EO $=2$ and $n_{\mathrm{d}}=2$ : they are both 


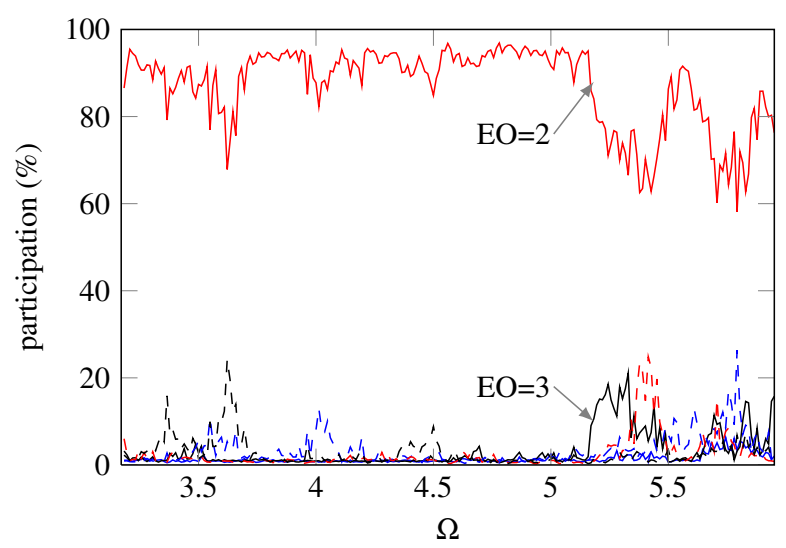

(a)

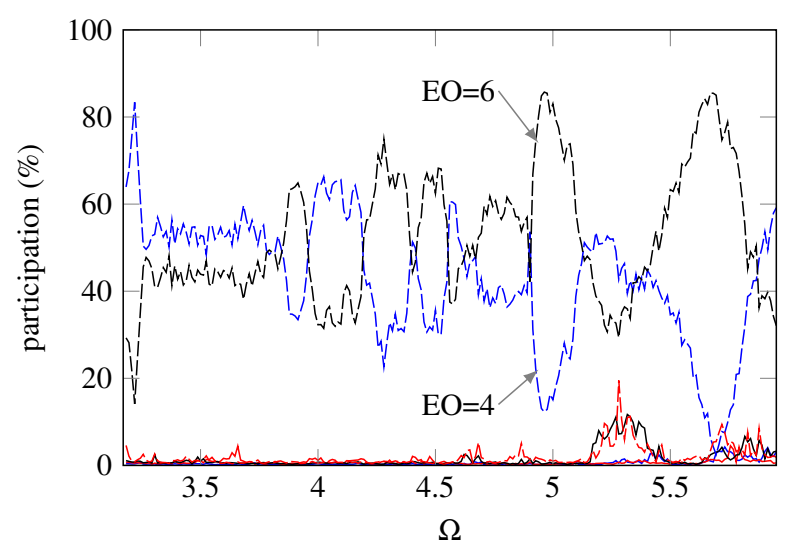

(b)

Figure 26. Engine order tracking: $\mathrm{EO}=1(-), \mathrm{EO}=2(-)$, $\mathrm{EO}=3(-), \mathrm{EO}=4(---), \mathrm{EO}=5(---)$ and $\mathrm{EO}=6(---)$, (a) $n_{\mathrm{d}}=2$, (b) $n_{\mathrm{d}}=4$

exclusively related meaning that $n_{\mathrm{d}}=2$ is only found along $E O=2$ and $E O=2$ only contains $n_{\mathrm{d}}=2$. Conversely, nodal diameter $n_{\mathrm{d}}=4$ combines $\mathrm{EO}=4$ and $\mathrm{EO}=6$ for the entire angular velocity range. Interestingly, critical angular velocities yielding vibratory resonances are detected in Figure 22 are in line with those for which $\mathrm{EO}=6$ dominates $\mathrm{EO}=4$ in Figure 26(b).

\subsection{Interaction for a three-lobe distorted casing}

The second interaction of interest features three privileged contact areas as depicted in Figure 21(b). Similarly to the two-lobe case, the interaction map for the full model is broken down into nodal diameter participations, as depicted in Figure 27.

Since EO $=3$ is smaller than the maximum number of nodal diameters featured by the impeller, a fundamental nodal diameter $n_{\mathrm{d}}=3$ is expected from Figure 13. Additionally, nodal diameters $n_{\mathrm{d}}=1$ and $n_{\mathrm{d}}=4$ are expected to feature non negligible amplitudes of vibration along engine orders $\mathrm{EO}=9$ and $\mathrm{EO}=6$ respectively, due to the aliasing effect. These predictions are explicitely supported by Figure 27 . Noticeably, peaks of large amplitude are not all detected along the main $\mathrm{EO}=3$ but also along $\mathrm{EO}=6$ and $\mathrm{EO}=9$, strongly connected to the four and one-nodal diameter free vibration modes. The fact that the impeller response to a three-lobe contact configuration involves four-nodal diameter free vibration modes may look counter intuitive and stresses the difficulty to accurately predict interactions based on condition (2).

Associated contact areas maps are plotted in Figs. 28(a) and. 28(b) at the leading edge and the trailing edge of the first

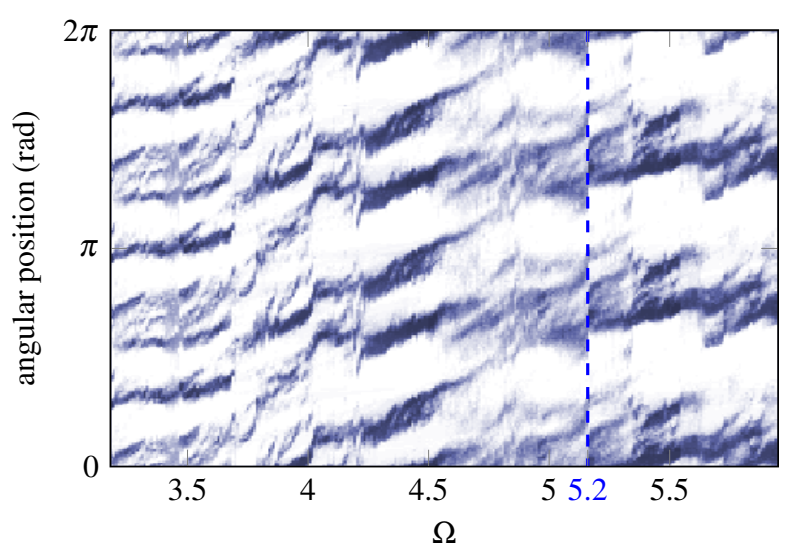

(a)

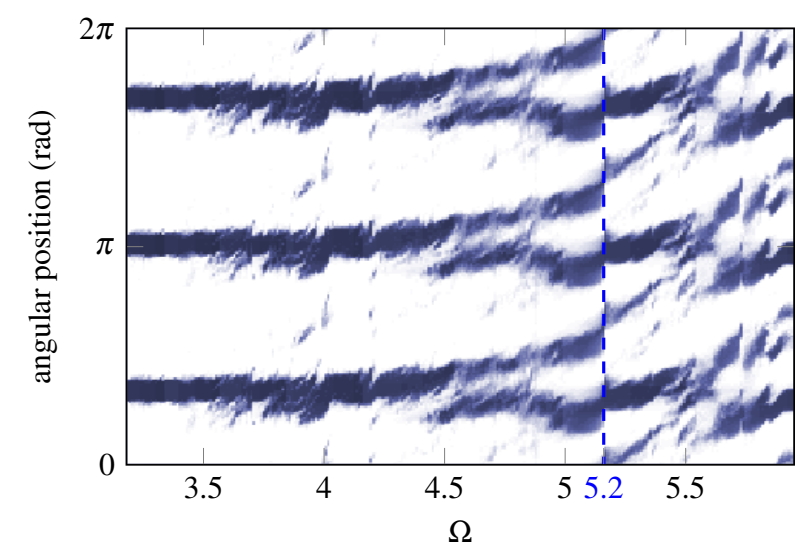

(b)

Figure 28. Contact areas maps, (a) leading edge, (b) trailing edge

sector blade, respectively. Similarly to what was observed with an ovalized casing, a more flexible leading edge manifests itself with low contrast contact maps while a very stiff trailing edge exhibits clearly identified contact areas. In the spirit of bifurcation diagrams, the abrupt transition in Figure 28(b) at $\Omega=5.2$ seems of particular interest: a small shift in the angular velocity leads to an radical change of the contact pattern on the casing. This suggests that the system may bifurcate from a stable solution to another, as previously observed for axial compressor blades in the vicinity of a critical speed [26].

\section{Conclusions}

Based on an existing time-marching numerical tool devoted to unilateral contact induced rotor/stator interactions in turbomachines, this works details a general methodology dedicated to the thorough analysis and comprehension of the dynamics of a bladed-disk assembly rotating within a statically distorted rigid casing. Centrifugal stiffening is included in the proposed reducedorder model. Cyclically symmetric structures and their key features such as aliasing and frequency clustering are recalled and systematically exploited within a post-processing methodology in the form of two-dimensional Fourier transforms, harmonic tracking, interaction maps and contact areas maps. Specific considerations regarding the aliasing phenomenon and its implication on the interpretation of the time harmonics of the contact forcing are extensively detailed. The space-time coupling of the contact forcing induced by the perfectly rigid casing allows for an explicit mapping of the time harmonics onto the nodal diameters of the impeller. 


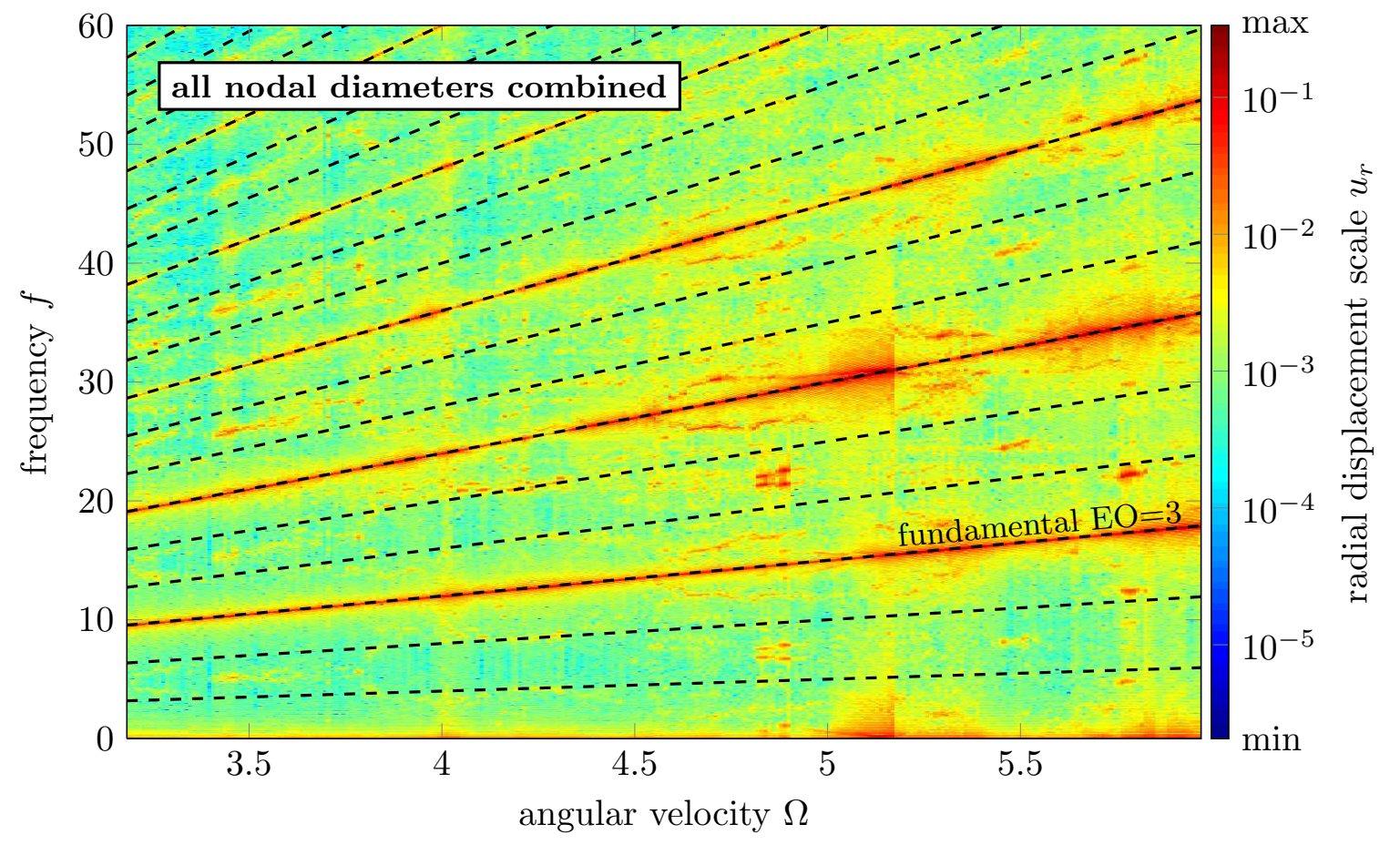

(a)
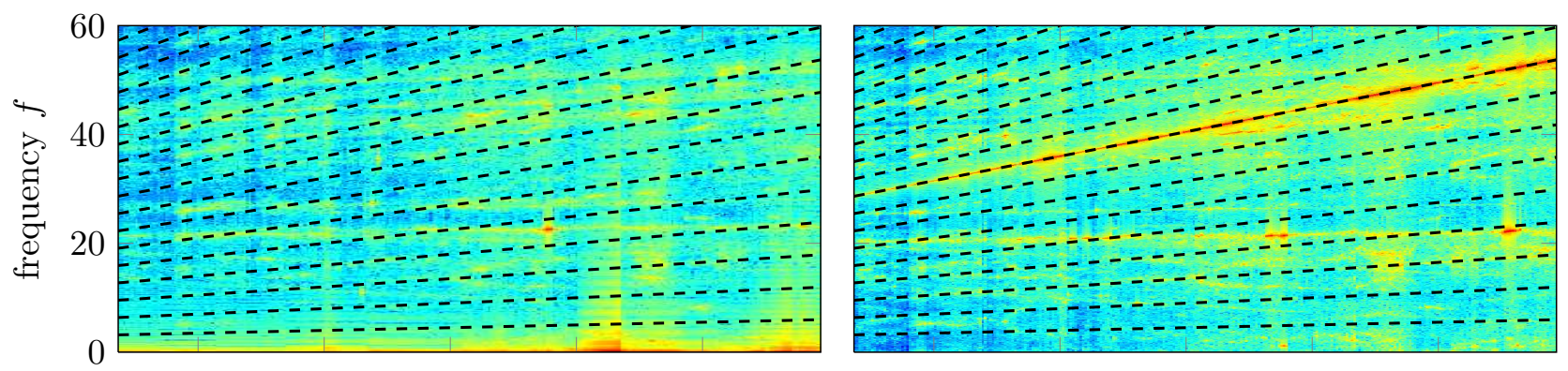

(b)

(c)
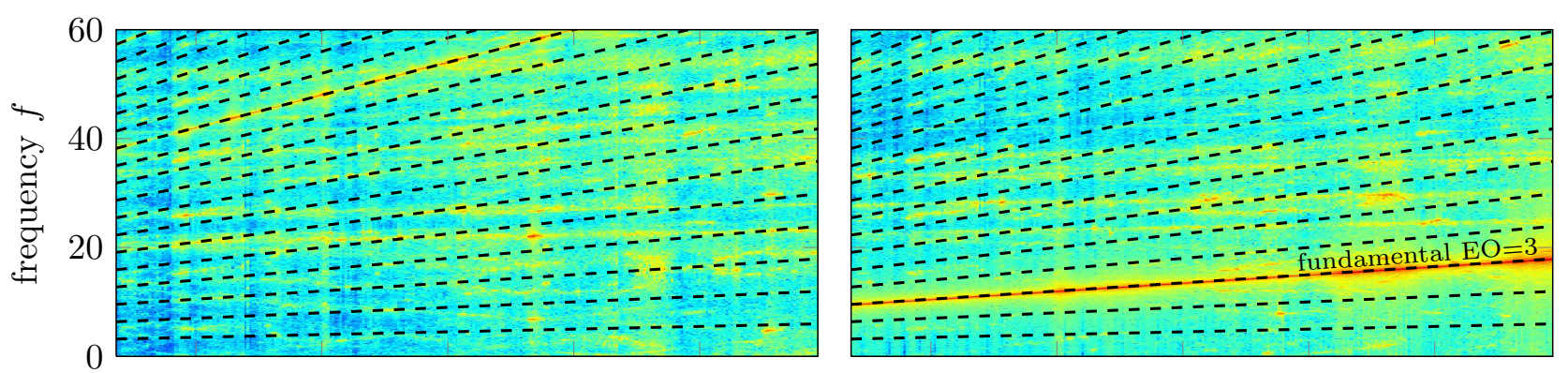

(d)

(e)
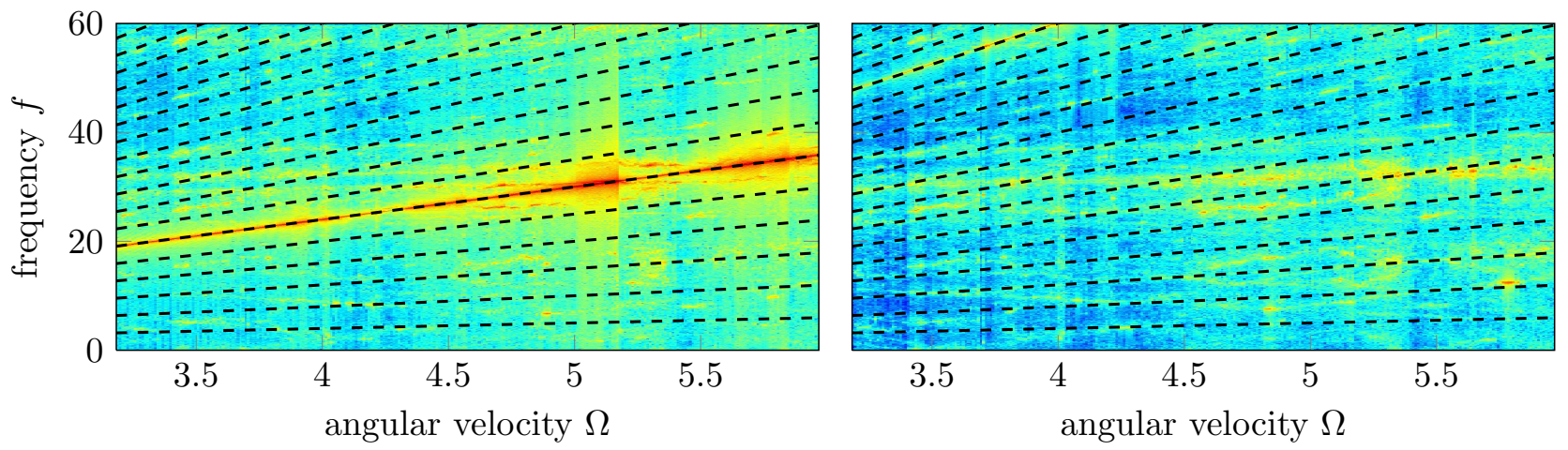

(f)

(g)

Figure 27. Interaction maps for a 3-lobe casing distortion, (a) interaction map, (b) $n_{\mathrm{d}}=0$, (c) $n_{\mathrm{d}}=1$, (d) $n_{\mathrm{d}}=2$, (e) $n_{\mathrm{d}}=3$, (f) $n_{\mathrm{d}}=4$, (g) $n_{\mathrm{d}}=5$ 
The proposed analysis is then deployed on two configurations of interest involving the 3D finite element model of an impeller. The presented results underline the fundamental role of superharmonics in the vibratory response of the impeller stemming from the highly nonlinear nature of the unilateral contact forcing. It is suggested that most detected critical interaction velocities may not be predicted by the commonly accepted criteria based on results obtained with a linear model applied to a rigid casing configuration.

The presented work is thus a first step toward the definition of a new methodology for the definition of nonlinear criteria suitable for enhancing the conception phase of impellers. It is advantageously applicable to any type of impeller, from large scale radial compressors or automotive turbochargers to microrotors.

\section{Acknowledgement}

Thanks go to Turbomeca and Snecma for their technical and financial support.

\section{A. Aliasing effect: analytical example}

The following one-dimensional equation of motion of the elementary sector $i$ of a cyclically symmetric structure is considered:

$$
m \ddot{x}_{i}(t)+k x_{i}(t)=f_{i}(t)
$$

It is now assumed that the cyclically symmetric structure possesses 10 elementary sectors, $i=1, \ldots, 10$ of angular span $\alpha=2 \pi / 10$ : the maximum number of nodal diameters $n_{\mathrm{d}}$ is $\lfloor 10 / 2\rfloor=5$. A 2 D-Fourier transform of $x_{i}(t), i=1, \ldots, 10$ yields:

$x_{i}(t)=\sum_{j=1}^{\infty} \sum_{n_{\mathrm{d}}=0}^{5}\left(x_{j, n_{\mathrm{d}}}^{c} \cos \left(j p \omega t+i n_{\mathrm{d}} \alpha\right)+x_{j, n_{\mathrm{d}}}^{s} \sin \left(j p \omega t+i n_{\mathrm{d}} \alpha\right)\right.$

For the sake of simplicity, only the fundamental harmonic $(j=$ 1) is considered and Eq. (19) reduces to:

$x_{i}(t)=\sum_{n_{\mathrm{d}}=0}^{5}\left(x_{1, n_{\mathrm{d}}}^{c} \cos \left(p \omega t+i n_{\mathrm{d}} \alpha\right)+x_{1, n_{\mathrm{d}}}^{s} \sin \left(p \omega t+i n_{\mathrm{d}} \alpha\right)\right)$

The forcing $f_{i}(t)$ on sector $i$ is assumed to be:

$f_{i}(t)=F \cos (p(\omega t+i \alpha))$

Accordingly, all coefficients $x_{1, n_{\mathrm{d}}}^{s}=0, n_{\mathrm{d}}=0, \ldots, 5$ in Eq. (20). The remaining coefficients $x_{1, n_{\mathrm{d}}}^{c}$ are computed for different values of $p$ and are listed in Tab. 1. While $p$ is smaller than the

Table 1. Coefficients $x_{1, n_{\mathrm{d}}}^{c}$ for different values of $p$

\begin{tabular}{c|cccccc}
$\mathrm{p}$ & $x_{1,0}^{c}$ & $x_{1,1}^{c}$ & $x_{1,2}^{c}$ & $x_{1,3}^{c}$ & $x_{1,4}^{c}$ & $x_{1,5}^{c}$ \\
\hline 3 & 0 & 0 & 0 & $\frac{F}{k-9 m \omega^{2}}$ & 0 & 0 \\
6 & 0 & 0 & 0 & 0 & $\frac{F}{k-36 m \omega^{2}}$ & 0 \\
9 & 0 & $\frac{F}{k-81 m \omega^{2}}$ & 0 & 0 & 0 & 0 \\
12 & 0 & 0 & $\frac{F}{k-144 m \omega^{2}}$ & 0 & 0 & 0 \\
\hline
\end{tabular}

maximum number of nodal diameter, only the coefficient $x_{1}^{c}$ is non zero. For higher values of $p$, it is found that only one coefficient $x_{1, n_{\mathrm{d}}}^{c}$ is non zero. This coefficient is said to be aliased and the relationship between $p$ and $n_{\mathrm{d}}$ is predicted by Figure 13

\section{References}

[1] W.-H. Jeon, D.-J. Lee, An analysis of the flow and aerodynamic acoustic sources of a centrifugal impeller, Journal of Sound and Vibration 222 (1999) 505-511.

[2] T. Raitor, W. Neise, Sound generation in centrifugal compressors, Journal of Sound and Vibration 314 (2008) 738-756.

[3] C. Scheit, B. Karic, S. Becker, Effect of blade wrap angle on efficiency and noise of small radial fan impellers - a computational and experimental study, Journal of Sound and Vibration 331 (2012) 996-1010.

[4] W.-M. Zhang, G. Meng, Contact dynamics between the rotor and bearing hub in an electrostatic micromotor, Microsystem technologies 11 (2005) 438-443.

[5] W.-M. Zhang, G. Meng, D. Chen, J.-B. Zhou, J.-Y. Chen, Nonlinear dynamics of a rub-impact micro-rotor system with scale-dependent friction model, Journal of Sound and Vibration 309 (2008) 756-777.

[6] B. Schweizer, Total instability of turbocharger rotors - physical explanation of the dynamic failure of rotors with fullfloating ring bearings, Journal of Sound and Vibration 328 (2009) 156-190.

[7] B. Schweizer, M. Sievert, Nonlinear oscillations of automotive turbocharger turbines, Journal of Sound and Vibration 321 (2009) 955-975.

[8] C. L. Morfey, Rotating pressure patterns in ducts: their generation and transmission, Journal of Sound and Vibration 1 (1) (1964) 60 - 87. doi : 10.1016/0022-460X (64) 90007-0.

[9] J. C. Wilkes, D. W. Childs, B. J. Dyck, S. G. Phillips, The numerical and experimental characteristics of multimode dry-friction whip and whirl, Journal of Engineering for Gas

$\left.n_{\mathrm{d}} \alpha\right)$ ) Turbines and Power 132 (5). doi : 10.1115/1. 3204658.

[10] D. W. Childs, D. Kumar, Dry-friction whip and whirl predictions for a rotor-stator model with rubbing contact at two locations, Journal of Engineering for Gas Turbines and Power 134 (7). doi : 10.1115/1.4005979.

[11] M. Legrand, A. Batailly, B. Magnain, P. Cartraud, C. Pierre, Full three-dimensional investigation of structural contact interactions in turbomachines, Journal of Sound and Vibration 331 (11) (2012) 2578-2601.

[12] A. Batailly, M. Legrand, A. Millecamps, F. Garcin, Numerical-experimental comparison in the simulation of rotor/stator interaction through blade-tip/abradable coating contact, Journal of Engineering for Gas Turbines and Power 134 (8).

[13] A. Millecamps, J. Brunel, P. Dufrénoy, F. Garcin, M. Nucci, Influence of thermal effects during blade-casing contact experiments, in: Proceedings of the ASME 2009 IDETC \& CIE Conference, ASME, 2009.

[14] R. J. Williams, Simulation of blade casing interaction phenomena in gas turbines resulting from heavy tip rubs using an implicit time marching method, in: Proceedings of the ASME Turbo Expo 2011, GT2011-45495, Vancouver, Canada, 2011. 
[15] N. White, S. Laney, C. Zorzi, Rcfa for recurring impeller failures in a $4.7 \mathrm{mtpa}$ lng train propane compressor, in: Proceedings of the Fortieth Turbomachinery Synposium, Houston, USA, 2011.

[16] C. Pierre, D. V. Murthy, Aeroelastic modal characteristics of mistuned blade assemblies: mode localization and loss of eigenstructure, AIAA Journal 30 (10) (1992) 2483 - 2496.

[17] P. D. Cha, C. Pierre, Eigensolution of periodic assemblies of multi-mode component systems, Journal of Sound and Vibration 129 (1) (1989) 168-174.

[18] M. B. Meingast, A. Batailly, M. Legrand, J. P. Ousty, Investigation of rotor-casing interactions in the centrifugal compressor of a helicopter engine, in: Proceedings of the ASME Turbo Expo 2013, GT2013-94461, San Antonio, USA, 2013.

[19] D. Thomas, Dynamics of rotationally periodic structures, International Journal for Numerical Methods in Engineering 14 (1) (1979) 81-102.

[20] R. Bladh, Efficient predictions of the vibratory response of mistuned bladed disks by reduced order modeling, Ph.d. thesis, The University of Michigan, Ann Arbor, USA (2001).

[21] A. Sternchüss, E. Balmès, On the reduction of quasi-cyclic disks with variable rotation speeds, Proceedings of the Inter- national Conference on Advanced Acoustics and Vibration Engineering (ISMA) (2006) 3925-3939.

[22] R. Craig, M. Bampton, Coupling of substructures for dynamic analyses, AIAA Journal 6 (7) (1968) 1313-1319.

[23] N. Carpenter, R. Taylor, M. Katona, Lagrange constraints for transcient finite element surface contact, International Journal for Numerical Methods in Engineering 32 (1991) 103-128.

[24] D. Jiang, C. Pierre, S. W. Shaw, Large-amplitude non-linear normal modes of piecewise linear systems, Journal of Sound and Vibration 272 (3-5) (2006) 869-891.

[25] A. Batailly, M. Legrand, A. Millecamps, S. Cochon, F. Garcin, Redesign of a high-pressure compressor blade accounting for nonlinear structural interactions, in: Proceedings of the ASME Turbo Expo 2014, GT2014-25673, Dusseldorf, Germany, 2014.

[26] A. Batailly, Legrand, Conjectural bifurcation analysis of an aircraft engine blade undergoing $3 \mathrm{~d}$ unilateral contact constraints, in: Proceedings of the ASME Turbo Expo 2014, GT2014-25674, Dusseldorf, Germany, 2014. 